\title{
ENTROPY DISSIPATIVE ONE-LEG MULTISTEP TIME APPROXIMATIONS OF NONLINEAR DIFFUSIVE EQUATIONS
}

\author{
ANSGAR JÜNGEL AND JOSIPA-PINA MILIŠIĆ
}

\begin{abstract}
New one-leg multistep time discretizations of nonlinear evolution equations are investigated. The main features of the scheme are the preservation of the nonnegativity and the entropy-dissipation structure of the diffusive equations. The key ideas are to combine Dahlquist's G-stability theory with entropy-dissipation methods and to introduce a nonlinear transformation of variables which provides a quadratic structure in the equations. It is shown that G-stability of the one-leg scheme is sufficient to derive discrete entropy dissipation estimates. The general result is applied to a cross-diffusion system from population dynamics and a nonlinear fourth-order quantum diffusion model, for which the existence of semi-discrete weak solutions is proved. Under some assumptions on the operator of the evolution equation, the second-order convergence of solutions is shown. Moreover, some numerical experiments for the population model are presented, which underline the theoretical results.
\end{abstract}

\section{INTRODUCTION}

Evolution equations with applications in the natural sciences typically contain some structural information reflecting inherent physical properties such as positivity, mass conservation, or energy and entropy dissipation. In this paper, we propose novel one-step and two-step semidiscrete numerical schemes, which preserve the structure of the underlying diffusive equations. For the analysis, we combine linear multistep discretizations, investigated for ordinary differential equations from the 1980s on, and entropy dissipation methods, which have been proposed in recent years.

Linear multistep methods refer to previous time steps and derivative values. They are proposed to solve stiff differential equations. An important class of these methods are the backward differentiation formulas (BDF). Multistep methods were also applied to nonlinear evolution equations. For instance, linear multistep schemes for fully nonlinear problems, which are governed by a nonlinear mapping with sectorial first Fréchet derivative, were dealt with, by linearization, in [24, 38], and quasilinear evolution equations were

Date: March 9, 2021.

2000 Mathematics Subject Classification. 65M12, 35Q40, 92D25, 82D37.

Key words and phrases. Linear multistep methods, entropy dissipation, diffusion equations, population dynamics, quantum drift-diffusion equation, Derrida-Lebowitz-Speer-Spohn equation, existence of solutions.

The first author acknowledges partial support from the Austrian Science Fund (FWF), grants P22108, P24304, I395, and W1245, and the Austrian-French Project of the Austrian Exchange Service (ÖAD). This research was supported by the European Union under Grant Agreement number 304617 (MarieCurie Project "Novel Methods in Computational Finance"). 
treated in [36]. In [23, 37], multistep discretizations for problems governed by maximal monotone or monotone operators were studied. For monotone evolution equations, also other schemes were proposed, for instance, stiffly accurate implicit Runge-Kutta methods [19]. Error estimates for two-step BDF methods for nonlinear evolution equations were shown in [17, 18].

Dahlquist introduced in [10] so-called one-leg methods which need only one function evaluation in each time step. With every multistep method we can associate its oneleg counterpart and vice versa. It turned out that one-leg methods allow for a stability analysis for stiff nonlinear problems. The stability behavior was defined in terms of the socalled G-stability [11], which can be related to discrete "energy" dissipation [26]. Stiffness independent error estimates with the optimal order of convergence were derived in [28].

Our schemes are generalizations of dissipative multistep methods analyzed in, e.g., [12, [26, 27]. In order to explain the idea, we consider the evolution equation

$$
u_{t}+A(u)=0, \quad t>0, \quad u(0)=u_{0},
$$

where $A$ is some (nonlinear) operator defined on $D(A) \subset \mathcal{V}$, where $\mathcal{V} \hookrightarrow \mathcal{H} \hookrightarrow \mathcal{V}^{\prime}$ is a Gelfand tripel (see Section 2.3 for details). In the literature, usually the monotonicity condition

$$
\langle A(u), u\rangle \geq 0 \text { for all } u \in D(A),
$$

where $\langle\cdot, \cdot\rangle$ is the dual product between $\mathcal{V}^{\prime}$ and $\mathcal{V}$, is assumed [26, 28]. This condition implies that the "energy" $\frac{1}{2}\|u(t)\|^{2}$ is nonincreasing:

$$
\frac{1}{2} \frac{d}{d t}\|u(t)\|^{2}=\left\langle u_{t}(t), u(t)\right\rangle=-\langle A(u(t)), u(t)\rangle \leq 0
$$

where $\|\cdot\|$ is the norm on $\mathcal{H}$.

In many situations, not the "energy" is increasing but a nonlinear expression $H[u(t)]$, which we call an "entropy". To explain this statement, we identify the Fréchet derivative $H^{\prime}[u]$ with its Riesz representative $h^{\prime}(u)$, i.e. $H^{\prime}[u] v=\left(h^{\prime}(u), v\right)$, where $(\cdot, \cdot)$ is the scalar product on $\mathcal{H}$. Then, replacing assumption (2) by

$$
\left\langle A(u), h^{\prime}(u)\right\rangle \geq 0 \quad \text { for all } u \in D(A),
$$

we find that formally

$$
\frac{d}{d t} H[u(t)]=\left(u_{t}(t), h^{\prime}(u(t))\right)=-\left\langle A(u(t)), h^{\prime}(u(t))\right\rangle \leq 0,
$$

i.e., $H[u]$ is a Lyapunov functional for (11) which expresses the dissipation property of the evolution equation. Note that condition (44) formally reduces to (2) for the special choice $H[u]=\frac{1}{2}\|u\|^{2}$.

In order to recover property (3) on a discrete level, Hill [26] discretizes (11) by the one-leg method

$$
\tau^{-1} \rho(E) u_{k}+A\left(\sigma(E) u_{k}\right)=0,
$$


where $u_{k}$ approximates $u\left(t_{k}\right)$ with $t_{k}=\tau k, \tau>0$ is the time step size, and

$$
\tau^{-1} \rho(E) u_{k}=\tau^{-1} \sum_{j=0}^{p} \alpha_{j} u_{k+j}, \quad \sigma(E) u_{k}=\sum_{j=0}^{p} \beta_{j} u_{k+j}
$$

with $\alpha_{j}, \beta_{j} \in \mathbb{R}$ are approximations of $u_{t}\left(t_{k}\right)$ and $u\left(t_{k}\right)$, respectively. Hill proves that, under some assumptions, this scheme yields a dissipative discretization if and only if the scheme is strongly A-stable or strongly G-stable (see Section 3.1 for a definition of G-stability). The proof relies on the quadratic structure of the definition of G-stability; this structure is already present in (3). Unfortunately, (5) does not possess such a structure and the proofs of [26] do not apply under the assumption (44).

The main idea of this paper is to enforce a quadratic structure by introducing the variable $v$ by $v^{2}=h(u)$ (assuming that $h(u) \geq 0$ ). More precisely, we discretize (10) in the formulation

$$
h(u)^{1 / 2} h^{\prime}(u)^{-1} v_{t}+\frac{1}{2} A(u)=0, \quad t>0, \quad v(0)=h\left(u_{0}\right)^{1 / 2},
$$

which is formally equivalent to (1). The semidiscrete scheme reads as

$$
h\left(w_{k}\right)^{1 / 2} h^{\prime}\left(w_{k}\right)^{-1} \rho(E) v_{k}+\frac{\tau}{2} A\left(w_{k}\right)=0, \quad w_{k}=h^{-1}\left(\left(\sigma(E) v_{k}\right)^{2}\right), \quad k \geq 0 .
$$

Note that $v_{k}$ approximates $h\left(u\left(t_{k}\right)\right)^{1 / 2}$ and $w_{k}$ approximates $u\left(t_{k}\right)$.

The first aim of this paper is to prove that this scheme dissipates the discrete entropy $H\left[V_{k}\right]$ with $V_{k}=\left(v_{k}, \ldots, v_{k+p-1}\right)$ (see Proposition [5), i.e.,

$$
H\left[V_{k+1}\right]-H\left[V_{k}\right] \leq 0,
$$

which is the discrete analogue of (5). Here, $H\left[V_{k}\right]$ is defined by

$$
H\left[V_{k}\right]=\frac{1}{2} \sum_{i, j=0}^{p-1} G_{i j}\left(v_{k+i}, v_{k+j}\right),
$$

and $G=\left(G_{i j}\right)$ is the matrix occuring in the definition of the G-stability (see Section 3 for details). Note that $H\left[V_{k}\right] \geq 0$ since $G$ is assumed to be positive definite.

The second aim of this paper is to prove that scheme (8) possesses an (entropy-dissipating) nonnegative weak solution. For the existence proof we need additional assumptions on the operator $A$. The main conditions are that equation (11) is nonnegativity-preserving and possesses two entropies, $h(u)=u^{\alpha}$ for $1<\alpha<2$ and $h(u)=u \log u$ (assuming that these expressions are defined). The nonnegativity-preservation of our scheme is inherited by the first condition. This property is proved by using the entropy density $h(u)=u \log u$ and the variable transformation $u=e^{y}>0$. The entropy density $h(u)=u^{\alpha}$ allows us to show the entropy-dissipation of our scheme. We believe that the condition $1<\alpha<2$ is technical. It is needed to control the discrete time derivative in (8) when using the test function $\log w_{k}$ (see Lemma 7).

A general existence proof would require more assumptions on $A$ which might restrict the applicability of our results. Therefore, we prefer to demonstrate the flexibility of our ideas 
by investigating two very different examples for $A$. The first example is a cross-diffusion system, the second one is a highly nonlinear equation of fourth order. In particular, our scheme is not restricted to scalar or second-order diffusion equations. For these examples, which are detailed in Sections 2.1 and 2.2, we give rigorous proofs of the existence of semidiscrete weak solutions to scheme (8).

Our third aim is to prove the second-order convergence of the one-leg scheme (8). Given a sequence of positive solutions $\left(v_{k}\right)$ to (8) and a smooth positive solution $v=u^{\alpha / 2}$ to (7), this means that there exists $C>0$ such that for all sufficiently small $\tau>0$,

$$
\left\|v_{k}-v\left(t_{k}\right)\right\| \leq C \tau^{2}, \quad t_{k}=\tau k, k \geq 1 .
$$

For this result, we assume that the mapping $v \mapsto v^{1-\alpha / 2} A\left(v^{2 / \alpha}\right)$ satisfies a one-sided Lipschitz condition and that the scheme $(\rho, \sigma)$ is of second order (see Theorem 3 for details). For instance, if $A$ is the fourth-order operator of the second example and $\alpha=1$, this assumption is satisfied [34]. The proof of (10) is similar to the proof of [25, Theorem V.6.10], based on an idea of Hundsdorfer and Steininger [28]. For convenience, we present the full proof, specialized to the present situation in general Hilbert spaces.

The paper is organized as follows. In Section 2, we state the main results on the existence of semidiscrete weak solutions and the convergence rate. General one-leg multistep schemes, which dissipate the energy or entropy, are discussed in Section 3 . Section 4 is devoted to the existence analysis. We detail the strategy of the existence proof in a general context and prove Theorems 1 and 2. The second-order convergence of the scheme is proved in Section 5. Numerical examples in Section 6 for the cross-diffusion population model, using the two-step BDF and the so-called $\gamma$-method (see Remark 4), show that the discrete entropy $H\left[V_{k}\right]$ decays exponentially fast to the stationary state. Finally, in the Appendix, we derive the family of all G-stable second-order one-leg schemes.

\section{MAin RESUlts}

We state the existence theorems for the semidiscretized cross-diffusion system and fourthorder equation and a theorem on the second-order convergence of the one-leg scheme.

2.1. Cross-diffusion population system. The first example is the cross-diffusion population model of Shigesada, Kawasaki, and Teramoto [39]:

$$
\begin{aligned}
& u_{t}^{(1)}-\operatorname{div}\left(\left(d_{1}+a_{1} u^{(1)}+u^{(2)}\right) \nabla u^{(1)}+u^{(1)} \nabla u^{(2)}\right)=0, \\
& u_{t}^{(2)}-\operatorname{div}\left(\left(d_{2}+a_{2} u^{(2)}+u^{(1)}\right) \nabla u^{(2)}+u^{(2)} \nabla u^{(1)}\right)=0, \quad t>0,
\end{aligned}
$$

where $u^{(j)}(x, t)$ is the density of the $j$-th species, $d_{1}, d_{2}>0$ are the diffusion coefficients, $a_{1}$, $a_{2}>0$ denote the self-diffusion coefficients, and the expression $\nabla\left(u^{(1)} u^{(2)}\right)=u^{(1)} \nabla u^{(2)}+$ $u^{(2)} \nabla u^{(1)}$ is the cross-diffusion term. The above system has been scaled in such a way that the coefficient of the cross-diffusion term is equal to one (see [20] for details). The equations are solved on the $d$-dimensional torus $\mathbb{T}^{d}$ with the initial conditions

$$
u^{(1)}(0)=u_{0}^{(1)}, \quad u^{(2)}(0)=u_{0}^{(2)} \quad \text { in } \mathbb{T}^{d} .
$$


Our results are also valid for homogeneous Neumann boundary conditions and suitable reaction (Lotka-Volterra) terms. This model describes the time evolution of two competing species neglecting Lotka-Volterra terms and effects of the environment. The basic idea is that the primary cause of dispersal is migration to avoid crowding instead of just random motion, modeled by diffusion. The model can be derived from a random walk on a lattice by assuming that the transition probabilities for a one-step jump depend linearly on the species' numbers.

The main feature of system (11)-(12) is that its diffusion matrix is generally neither symmetric nor positive definite. Using entropy methods, the implicit Euler time discretization and a partial finite-difference approximation, the global existence of weak solutions was shown in [7]. Instead of discretizing the cross-diffusion term by finite differences, an elliptic regularization was employed in [8]. Another (simpler) regularization was suggested in the finite-element context by Barrett and Blowey [3] by using an approximate entropy functional. For the one-dimensional equations, a temporally semi-discrete approximation was investigated in [20]. Andreianov et al. [1] employed a finite-volume method assuming positive definite diffusion matrices. A deterministic particle method with a Peaceman-Rachford operator splitting in time was developed by Gambino et al. 21]. In all these approaches (except [21]), an implicit Euler discretization was used. We allow for (G-stable) higher-order time discretizations.

We choose the entropy density $h(u)=\left(u^{(1)}\right)^{\alpha}+\left(u^{(2)}\right)^{\alpha}$ for $\alpha>1$ and $u=\left(u^{(1)}, u^{(2)}\right)$ and the discrete entropy

$$
H\left[V_{k}\right]=\frac{1}{2} \sum_{i, j=0}^{p-1} G_{i j} \int_{\mathbb{T}^{d}} v_{k+i} \cdot v_{k+j} d x=\frac{1}{2} \sum_{i, j=0}^{p-1} G_{i j} \int_{\mathbb{T}^{d}}\left(v_{k+i}^{(1)} v_{k+j}^{(1)}+v_{k+i}^{(2)} v_{k+j}^{(2)}\right) d x .
$$

Theorem 1 (Semidiscrete population system). Let $d \leq 3,1<\alpha<2$, and $4 a_{1} a_{2} \geq$ $\max \left\{a_{1}, a_{2}\right\}+1$. Let $v_{0}, \ldots, v_{p-1} \in L^{2}\left(\mathbb{T}^{d}\right)^{2}$ be nonnegative componentwise. Furthermore, let the scheme $(\rho, \sigma)$, defined in (6) , be G-stable (hence, $p \leq 2)$ and assume that $\alpha_{p}>0$ and $\beta_{p}>0$. Then there exists a sequence of weak solutions $\left(v_{k}, w_{k}\right)=\left(v_{k}^{(1)}, v_{k}^{(2)}, w_{k}^{(1)}, w_{k}^{(2)}\right) \in$ $L^{2}\left(\mathbb{T}^{d}\right)^{2} \times W^{1,3 / 2}\left(\mathbb{T}^{d}\right)^{2}$ to

$$
\begin{aligned}
& \frac{2}{\alpha \tau}\left(w_{k}^{(1)}\right)^{1-\alpha / 2} \rho(E) v_{k}^{(1)}+\operatorname{div}\left(\left(d_{1}+a_{1} w_{k}^{(1)}+w_{k}^{(2)}\right) \nabla w_{k}^{(1)}+w_{k}^{(1)} \nabla w_{k}^{(2)}\right)=0, \\
& \frac{2}{\alpha \tau}\left(w_{k}^{(2)}\right)^{1-\alpha / 2} \rho(E) v_{k}^{(2)}+\operatorname{div}\left(\left(d_{2}+a_{2} w_{k}^{(2)}+w_{k}^{(1)}\right) \nabla w_{k}^{(2)}+w_{k}^{(2)} \nabla w_{k}^{(1)}\right)=0,
\end{aligned}
$$

where $w_{k}^{(j)}=\left(\sigma(E) v_{k}^{(j)}\right)^{2 / \alpha}, \sigma(E) v_{k}^{(j)} \geq 0$, and $w_{k}^{(j)} \in L^{3 \alpha}\left(\mathbb{T}^{d}\right)^{2}(j=1,2, k \geq 0)$. The scheme dissipates the entropy in the sense

$$
H\left[V_{k+1}\right]+\frac{2 \tau}{\alpha^{2}}(\alpha-1) \int_{\mathbb{T}^{d}}\left(d_{1}\left|\nabla w_{1, k}^{\alpha / 2}\right|^{2}+d_{2}\left|\nabla w_{2, k}^{\alpha / 2}\right|^{2}\right) d x \leq H\left[V_{k}\right],
$$

where $H\left[V_{k}\right]$ is defined in (14).

The condition $4 a_{1} a_{2} \geq \max \left\{a_{1}, a_{2}\right\}+1$ is needed to prove that the cross-diffusion system (11)-(12) dissipates the entropy for $1<\alpha<2$; see Lemma 10 . 
2.2. Fourth-order quantum diffusion equation. We consider the quantum diffusion equation, also called Derrida-Lebowitz-Speer-Spohn (DLSS) equation,

$$
u_{t}+\nabla^{2}:\left(u \nabla^{2} \log u\right)=0, \quad t>0, \quad u(0)=u_{0} \quad \text { in } \mathbb{T}^{d},
$$

where $\nabla^{2} u$ is the Hessian matrix of $u$ and $A: B=\sum_{i, j} A_{i j} B_{i j}$ is the Frobenius inner product between matrices. This equation is the zero-temperature and zero-field limit of the quantum drift-diffusion model, which describes the evolution of the electron density $u(t)$ in a quantum semiconductor device [29]. It was derived in [14] from a relaxation-time Wigner equation. Its one-dimensional version was derived in [15] in a suitable scaling limit from the time-discrete Toom model, where $u$ is related to a random variable.

The global-in-time existence of nonnegative weak solutions to (18) was proven in [22, 31]. Most of the numerical schemes proposed for (18) are based on an implicit Euler discretization in one space dimension. In [33], the convergence of a positivity-preserving semidiscrete Euler scheme was shown. A fully discrete finite-difference scheme which preserves the positivity, mass, and physical entropy was derived in [6]. Düring et al. [16] employed the variational structure of (18) on a fully discrete level and introduced a discrete minimizing movement scheme. Finally, a two-step BDF method was applied to (18) in [4] and the second-order convergence of semidiscrete solutions was shown. Here, we generalize [4] by allowing for general (G-stable) second-order time discretizations.

We choose the entropy density $h(u)=u^{\alpha}$ for $\alpha>1$ and the discrete entropy $H\left[V_{k}\right]=$ $\frac{1}{2} \sum_{i, j=0}^{p-1} G_{i j} \int_{\mathbb{T}^{d}} v_{k+i} v_{k+j} d x$.

Theorem 2 (Semidiscrete DLSS equation). Let $1 \leq d \leq 3,1<\alpha<(\sqrt{d}+1)^{2} /(d+2)$, and let $v_{0}, \ldots, v_{p-1} \in L^{2}\left(\mathbb{T}^{d}\right)$ be nonnegative. Furthermore, let the scheme $(\rho, \sigma)$ be $G$-stable (hence, $p \leq 2$ ) and assume that $\alpha_{p}>0$ and $\beta_{p}>0$ hold. Then there exists a sequence of weak solutions $\left(v_{k}, w_{k}\right) \in L^{2}\left(\mathbb{T}^{d}\right) \times L^{\infty}\left(\mathbb{T}^{d}\right)$ to

$$
\frac{2}{\alpha \tau} w_{k}^{1-\alpha / 2} \rho(E) v_{k}+\nabla^{2}:\left(w_{k} \nabla^{2} \log w_{k}\right)=0,
$$

satisfying $w_{k}=\left(\sigma(E) v_{k}\right)^{2 / \alpha}, \sigma(E) v_{k} \geq 0, w_{k}^{\alpha / 2} \in H^{2}\left(\mathbb{T}^{d}\right)$, and $w_{k}^{1 / 2} \in W^{1,2 \alpha}\left(\mathbb{T}^{d}\right)$, in the following sense: For all $\phi \in W^{2, \alpha /(\alpha-1)}\left(\mathbb{T}^{d}\right)$ and $k \geq 0$,

$$
\frac{1}{\tau} \int_{\mathbb{T}^{d}} w_{k}^{1-\alpha / 2} \rho(E) v_{k} \phi d x+\int_{\mathbb{T}^{d}}\left(w_{k}^{1-\alpha / 2} \nabla^{2} w_{k}^{\alpha / 2}-\alpha^{2} \nabla w_{k}^{1 / 2} \otimes \nabla w_{k}^{1 / 2}\right): \nabla^{2} \phi d x=0 .
$$

The scheme dissipates the entropy in the sense

$$
H\left[V_{k+1}\right]+\frac{\alpha}{4} \kappa_{\alpha} \tau \int_{\mathbb{T}^{d}}\left(\Delta w_{k}^{\alpha / 2}\right)^{2} d x \leq H\left[V_{k}\right]
$$

where $\kappa_{\alpha}>0$ only depends on $\alpha$ and $d$.

Again, the condition $1<\alpha<(\sqrt{d}+1)^{2} /(d+2)$ is needed to derive the entropy dissipation of (18); see [31]. 
2.3. Second-order convergence rate. Let $\mathcal{V} \hookrightarrow \mathcal{H} \hookrightarrow \mathcal{V}^{\prime}$ be a Gelfand tripel [41, Section 23.4], where $\mathcal{V}$ is a Banach space and $\mathcal{H}$ is a Hilbert space with scalar product $(\cdot, \cdot)$ and norm $\|\cdot\|$. We assume that we can define the notion of positivity on $\mathcal{H}$. Furthermore, let $A: D(A) \rightarrow \mathcal{V}^{\prime}$ be a (nonlinear) operator with domain $D(A) \subset \mathcal{V}$ and let $h(u)=u^{\alpha}$ for $u \in D(A), u>0$ with $\alpha \geq 1$. (We assume that the expression $u^{\alpha}$ makes sense in $\mathcal{V}$.) Then, given $v_{0}=u_{0}^{\alpha / 2}$, let $v_{1}$ be the solution to the implicit Euler scheme (which is assumed to exist)

$$
\frac{2}{\alpha \tau}\left(v_{1}-v_{0}\right)+v_{1}^{1-2 / \alpha} A\left(v_{1}^{2 / \alpha}\right)=0 .
$$

We assume that the scheme $(\rho, \sigma)$ with $p=2$ is G-stable and that the differentation error $\delta_{D}(t)$ and the interpolation error $\delta_{I}(t)$, defined by [25, Section V.6]

$$
\delta_{D}(t)=\rho(E) v(t)-\tau v_{t}(t+2 \tau), \quad \delta_{I}(t)=\sigma(E) v(t)-v(t+2 \tau)
$$

are of second order (see Section 3.1).

Theorem 3. Let $\left(v_{k}\right)$ be a sequence of smooth solutions to (8) and (21) satisfying $\sigma(E) v_{k}>$ 0 and let $u$ be a smooth positive solution to (1). Let the above assumptions on the scheme $(\rho, \sigma)$ hold. We assume that the mapping $v \mapsto B(v)=\frac{\alpha}{2} v^{1-2 / \alpha} A\left(v^{2 / \alpha}\right)$ is well defined and satisfies the one-sided Lipschitz condition

$$
\langle B(v)-B(\bar{v}), v-\bar{v}\rangle \geq-\kappa_{1}\|v-\bar{v}\|^{2} \quad \text { for all } v^{2 / \alpha}, \bar{v}^{2 / \alpha} \in D(A)
$$

for some $\kappa_{1}>0$. Then there exist $\tau_{0}>0$ and $C>0$ such that for all $0<\tau \leq \tau_{0}$,

$$
\left\|v_{k}-u\left(t_{k}\right)^{\alpha / 2}\right\| \leq C \tau^{2}, \quad t_{k}=\tau k, k \geq 0 .
$$

The one-sided Lipschitz condition is also needed in [25, Section V.6]. It is satisfied, for instance, for the operator of the population model (11)-(12) with domain contained in $W^{1, \infty}(\Omega)$, or for monotone operators $B$. We give some examples for the latter case. If $A: D(A) \rightarrow \mathcal{V}^{\prime}$ is any monotone operator, the assumption of the theorem is trivially satisfied for $\alpha=2$ since then $A=B$. In this situation, we recover the "energy" method described in the introduction.

Next, let $A: D \rightarrow H^{-2}(\Omega)$ with $A(w)=\nabla^{2}:\left(w \nabla^{2} \log w\right)$ for $w \in D=\left\{w \in H^{2}(\Omega)\right.$ : $w>0$ in $\Omega\}$ and $\Omega \subset \mathbb{R}^{d}(d \leq 3)$ be the operator of the DLSS equation. It is shown in [34, Lemma 3.5] that $v \mapsto v^{-1} A\left(\bar{v}^{2}\right)$ is monotone in the sense of $\left\langle v_{1}^{-1} A\left(v_{1}^{2}\right)-v_{2}^{-1} A\left(v_{2}^{2}\right), v_{1}-v_{2}\right\rangle \geq$ 0 for $v_{1}^{2}, v_{2}^{2} \in D$. This operator satisfies the assumptions of Theorem 3 for $\alpha=1$. In fact, the above theorem is a generalization of Theorem 2 in [4], which is proved for $\alpha=1$ and the two-step BDF method only. Another example is $\alpha=4 / 3$ and the fast-diffusion operator $A(u)=-\Delta\left(u^{1 / 3}\right)$ with Dirichlet boundary conditions, although we do not study this operator here.

\section{General one-Leg multistep schemes}

We wish to semi-discretize the Cauchy problem (1) in its weak formulation

$$
\left\langle u_{t}(t), \phi\right\rangle+\langle A(u(t)), \phi\rangle=0 \quad \text { for all } \phi \in \mathcal{V}, t>0, \quad u(0)=u_{0},
$$


where $\langle\cdot, \cdot\rangle$ is the dual product between $\mathcal{V}^{\prime}$ and $\mathcal{V}$ (see Section 2.3 for the notations). We assume that there exists a (smooth) solution $u:[0, T] \rightarrow D(A)$ to (23) .

3.1. One-leg schemes and energy dissipation. We recall some basic notions of one-leg schemes and G-stability. We introduce the time steps $t_{k}=\tau k$, where $\tau>0$ is the time step size and $k \in \mathbb{N}$. One-leg methods can be formulated in compact form by introducing the polynomials

$$
\rho(\xi)=\sum_{j=0}^{p} \alpha_{j} \xi^{j}, \quad \sigma(\xi)=\sum_{j=0}^{p} \beta_{j} \xi^{j}
$$

where $\alpha_{j}, \beta_{j} \in \mathbb{R}, \alpha_{p} \neq 0$, and we normalize $\sigma(1)=1$. Let $E u_{k}=u_{k+1}$ be the forward time shift, defined on the sequence $\left(u_{k}\right)$. Then

$$
\rho(E) u_{k}=\sum_{j=0}^{p} \alpha_{j} u_{k+j}, \quad \sigma(E) u_{k}=\sum_{j=0}^{p} \beta_{j} u_{k+j}, \quad k \geq 0 .
$$

As mentioned in the introduction, the standard one-leg discretization of (11) reads as

$$
\tau^{-1} \rho(E) u_{k}+A\left(\sigma(E) u_{k}\right)=0, \quad k \geq 0,
$$

where $u_{k}$ and $\sigma(E) u_{k}$ approximate $u\left(t_{k}\right)$ and $\tau^{-1} \rho(E) u_{k}$ approximates $u_{t}\left(t_{k}\right)$. The values $u_{0}, \ldots, u_{p-1}$ are assumed to be given.

According to [25, Exercise 1a, Section V.6], the conditions $\rho(1)=0, \rho^{\prime}(1)=\sigma(1)=1$ imply the consistency of the scheme $(\rho, \sigma)$. If additionally $\rho^{\prime}(1)+\rho^{\prime \prime}(1)=2 \sigma^{\prime}(1)$, the scheme is second-order accurate, i.e., the differentiation error (22) satisfies $\left\|\delta_{D}(t)\right\| \leq C_{D} \tau^{3}$ uniformly in $t \in(0, T)$. The constant $C_{D}>0$ depends on the $L^{\infty}(0, T ; \mathcal{H})$ norm of $v_{t t t}$. Furthermore, if $\sigma(1)=1$ and $\sigma^{\prime}(1)=2$, the interpolation error is of second order, i.e. $\left\|\delta_{I}(t)\right\| \leq C_{I} \tau^{2}$ uniformly in $t \in(0, T)$, and $C_{I}>0$ depends on $v_{t t}$.

Dahlquist [9] has proven that any A-stable scheme $(\rho, \sigma)$ is at most of second order. He related the discrete energy dissipation to a stability condition, called G-stability. We say that $(\rho, \sigma)$ is G-stable [26, Def. 2.4] if there exists a symmetric, positive definite matrix $G=\left(G_{i j}\right) \in \mathbb{R}^{p \times p}$ (called G-matrix in the following) such that for any sequence $\left(u_{k}\right)$ defined on $\mathcal{V}$,

$$
\left(\rho(E) u_{k}, \sigma(E) u_{k}\right) \geq \frac{1}{2}\left\|U_{k+1}\right\|_{G}^{2}-\frac{1}{2}\left\|U_{k}\right\|_{G}^{2} \quad \text { for all } k \in \mathbb{N},
$$

where the G-norm is given by

$$
\left\|U_{k}\right\|_{G}^{2}=\sum_{i, j=0}^{p-1} G_{i j}\left(u_{k+i}, u_{k+j}\right), \quad U_{k}=\left(u_{k}, \ldots, u_{k+p-1}\right) .
$$

Any scheme $(\rho, \sigma)$, for which $\rho(\xi)$ and $\sigma(\xi)$ are coprime polynomials, is G-stable if and only if it is A-stable [2, 11]. The proof in [2] provides constructive formulas for the matrix 
G (also see [25, Section V.6]). The G-stability and the monotonicity condition (2) imply energy dissipation since, formally, by (24),

$$
\frac{1}{2}\left\|U_{k}\right\|_{G}^{2}-\frac{1}{2}\left\|U_{k-1}\right\|_{G}^{2} \leq\left(\rho(E) u_{k}, \sigma(E) u_{k}\right)=-\tau\left(A\left(\sigma(E) u_{k}\right), \sigma(E) u_{k}\right) \leq 0 .
$$

In particular, the discrete energy $k \mapsto \frac{1}{2}\left\|U_{k}\right\|_{G}^{2}$ is nonincreasing. In the appendix, we derive all second-order one-leg schemes which are G-stable.

Remark 4. We give some known examples of G-stable one-leg methods. Examples (ii) and (iii) are included in the family of schemes derived in the appendix.

(i) The (first-order) implicit mid-point rule is defined by $p=1,\left(\alpha_{0}, \alpha_{1}\right)=(-1,1)$ and $\left(\beta_{0}, \beta_{1}\right)=\left(\frac{1}{2}, \frac{1}{2}\right)$. Then the G-norm coincides with the norm on $\mathcal{H}$.

(ii) The two-step BDF method is defined by $p=2,\left(\alpha_{0}, \alpha_{1}, \alpha_{2}\right)=\left(\frac{1}{2},-2, \frac{3}{2}\right)$ and $\left(\beta_{0}, \beta_{1}, \beta_{2}\right)$ $=(0,0,1)$. It is of second order and its G-matrix equals

$$
G=\frac{1}{2}\left(\begin{array}{cc}
1 & -2 \\
-2 & 5
\end{array}\right)
$$

(iii) A family of two-step one-leg methods is proposed in [12, 35] with $p=2$ and

$$
\begin{aligned}
\left(\alpha_{0}, \alpha_{1}, \alpha_{2}\right) & =\frac{1}{\gamma+1}(-\gamma, \gamma-1,1), \\
\left(\beta_{0}, \beta_{1}, \beta_{2}\right) & =\frac{1}{2(\gamma+1)^{2}}\left(\gamma(\gamma+3),(\gamma-1)^{2}, 3 \gamma+1\right),
\end{aligned}
$$

where $0<\gamma \leq 1$ is a free parameter. In [35], the value $\gamma=9-4 \sqrt{5} \approx 0.055$ is derived by optimizing the stability at infinity for this method, whereas the authors of [12] minimize the error constant of the method, which leads to $\gamma=1 / 5$. The scheme is of second order; the G-stability follows from the identity

$$
(\rho(E) u, \sigma(E) u)=U_{1}^{\top} G U_{1}-U_{0}^{\top} G U_{0}+\frac{1-\gamma}{2(\gamma+1)^{3}}\left(u_{0}-2 u_{1}+u_{2}\right)^{2}
$$

for all $u=\left(u_{0}, u_{1}, u_{2}\right)^{\top} \in \mathbb{R}^{3}$, where $U_{1}=\left(u_{1}, u_{2}\right)^{\top}, U_{0}=\left(u_{0}, u_{1}\right)^{\top}$, and the G-matrix

$$
G=\frac{1}{2(\gamma+1)}\left(\begin{array}{ll}
\gamma & 0 \\
0 & 1
\end{array}\right)
$$

is diagonal.

3.2. One-leg schemes and entropy dissipation. In this subsection, we introduce general one-leg schemes which dissipate the entropy. To this end, let $h: D(A) \rightarrow \mathcal{V}$ be a differentiable and invertible function. We assume that we can define the notion of nonnegativity on $\mathcal{V}$ and that $h(u) \geq 0$ for all $u \in D(A), u \geq 0$. Our main hypothesis is

$$
\left\langle A(\phi), h^{\prime}(\phi)\right\rangle \geq 0 \quad \text { for all } \phi \in D(A) .
$$

We have shown in (5) that this assumption implies that $H[u]$ is a Lyapunov functional for (1), where we identify the function $h^{\prime}(u)$ with the Fréchet derivative $H^{\prime}[u]$. Instead of 
discretizing (23) directly, we consider the weak formulation

$$
2\left(v_{t}, h(u)^{1 / 2} h^{\prime}(u)^{-1} \phi\right)+\langle A(u), \phi\rangle=0, \quad t>0, \quad v(0)=h\left(u_{0}\right)^{1 / 2},
$$

where $v=h(u)^{1 / 2}$, which is formally equivalent to (23). In order to be well defined, we assume that the product $h(u)^{1 / 2} h^{\prime}(u)^{-1} \phi$ is an element of $\mathcal{H}$ and that $u \geq 0$.

With the notations from Section 3.1, let $(\rho, \sigma)$ be a consistent scheme. Furthermore, let $u_{k}$ approximate $u\left(t_{k}\right)$ and define

$$
v_{k}=h\left(u_{k}\right)^{1 / 2}, \quad w_{k}=h^{-1}\left(\left(\sigma(E) v_{k}\right)^{2}\right), \quad k \in \mathbb{N},
$$

supposing that $\left(\sigma(E) v_{k}\right)^{2} \in \mathcal{V}$. Then $v_{k}$ approximates $h\left(u\left(t_{k}\right)\right)^{1 / 2}$ and $w_{k}$ is an approximation of $u\left(t_{k}\right)$. The one-leg multistep approximation of (27) is defined by

$$
\frac{2}{\tau}\left(\rho(E) v_{k}, h\left(w_{k}\right)^{1 / 2} h^{\prime}\left(w_{k}\right)^{-1} \phi\right)+\left\langle A\left(w_{k}\right), \phi\right\rangle=0, \quad w_{k}=h^{-1}\left(\left(\sigma(E) v_{k}\right)^{2}\right),
$$

where $k \geq 0$ and the values $v_{0}, \ldots, v_{p-1}$ are given. The existence of weak solutions is investigated in Section 4. The following proposition states that this scheme dissipates the discrete entropy

$$
H\left[V_{k}\right]=\frac{1}{2}\left\|V_{k}\right\|_{G}^{2}=\frac{1}{2} \sum_{i, j=0}^{p-1} G_{i j}\left(v_{k+i}, v_{k+j}\right), \quad V_{k}=\left(v_{k}, \ldots, v_{k+p-1}\right),
$$

if the scheme is G-stable.

Proposition 5. Let $(\rho, \sigma)$ be a G-stable scheme and assume that (26) holds. Let $\left(v_{k}\right)$ be a sequence of solutions to (28) such that $h^{\prime}\left(w_{k}\right) \in \mathcal{V}$. Then the scheme dissipates the discrete entropy, i.e., $k \mapsto H\left[V_{k}\right]$ is nonincreasing, where $V_{k}=\left(v_{k}, \ldots, v_{k+p-1}\right)$.

Proof. We employ the definition $h\left(w_{k}\right)^{1 / 2}=\sigma(E) v_{k}$ and the test function $\phi=h^{\prime}\left(w_{k}\right) \in \mathcal{V}$ in (28):

$$
\left(\rho(E) v_{k}, \sigma(E) v_{k}\right)=\left(\rho(E) v_{k}, h\left(w_{k}\right)^{1 / 2}\right)=-\frac{\tau}{2}\left\langle A\left(w_{k}\right), h^{\prime}\left(w_{k}\right)\right\rangle \leq 0 .
$$

Because of the G-stability (25), we find that for $k \geq 0$,

$$
H\left[V_{k+1}\right]-H\left[V_{k}\right]=\frac{1}{2}\left\|V_{k+1}\right\|_{G}^{2}-\frac{1}{2}\left\|V_{k}\right\|_{G}^{2} \leq\left(\rho(E) v_{k}, \sigma(E) v_{k}\right) \leq 0,
$$

finishing the proof.

Remark 6. Let $1 \in \mathcal{H},\left(u_{k}, 1\right)=\left(u_{0}, 1\right)$ for all $k=0, \ldots, p-1$, and $\left\langle A\left(\sigma(E) u_{k}\right), 1\right\rangle=$ 0 . Then scheme (24) preserve the mass, i.e., $\left(u_{k}, 1\right)=\left(u_{0}, 1\right)$ for all $k \in \mathbb{N}$. This is generally not the case for scheme (28). However, choosing $\phi=1$ in (28), it follows that $\left(\rho(E) v_{k}, h\left(w_{k}\right)^{1 / 2} h^{\prime}\left(w_{k}\right)^{-1}\right)=0$, which approximates $\partial_{t}(u(t), 1)=0$.

\section{ExistenCE OF SEMI-DisCRETE SOLUtions}

In this section, we first sketch the existence proof in the general situation (28) and explain the main ideas. Rigorous proofs are presented for the cross-diffusion system (11)-(12) in Section 4.2 and for the fourth-order quantum diffusion equation (18) in Section 4.3 . 
4.1. General strategy. The proof of the existence of weak solutions to scheme (28) is based on a regularization procedure and a fixed-point argument. In order to pass to the limit of vanishing regularization parameters, some compactness is needed, which strongly depends on the properties of the operator $A$ and the choice of the function spaces. Therefore, we only detail those parts of the proof which concern the time discretization and refer to the subsequent subsections for full proofs with specific examples of $A$ and the function spaces.

Let the assumptions at the beginning of Sections 2.3 and 3 hold and let $h(u)=u^{\alpha}$ with $1<\alpha<2$. Furthermore, let $\mathcal{H}=L^{2}(\Omega)$, where $\Omega \subset \mathbb{R}^{d}$ is some bounded domain. Let $D(A) \subset \mathcal{V}$ be a subspace such that $u^{\alpha-1}, \log u \in \mathcal{V}$ for all $u \in D(A)$ (examples are given in the following subsections). We suppose:

(i) The operator $A: D(A) \rightarrow \mathcal{V}^{\prime}$ satisfies $\left\langle A(u), u^{\alpha-1}\right\rangle \geq 0$ and $\langle A(u), \log u\rangle \geq 0$ for all $u \in D(A)$.

(ii) There exists a linear bounded operator $\widetilde{A}[z]: \mathcal{V} \rightarrow \mathcal{V}^{\prime}$ ("linearization" of $A$ around $z \in D(A))$ such that $\widetilde{A}\left[e^{y}\right](y)=A\left(e^{y}\right)$ for all $e^{y} \in D(A)$ and $\left\langle\widetilde{A}\left[e^{z}\right](y), y\right\rangle \geq 0$ for all $e^{z} \in D(A)$ and $y \in \mathcal{V}$.

The first condition in (i) corresponds to (26); the second condition is needed to derive uniform estimates in the variable $\log u$ for the fixed-point argument. Assumption (ii) is used to apply the Lax-Milgram lemma.

Step 1: Exponential variable transformation. First, we formulate scheme (28) in the variable $y=\log w_{k}$. This transformation provides the positivity of the numerical solution $w_{k}=e^{y}$. Let $k \in \mathbb{N}_{0}$ and $v_{k}, \ldots, v_{k+p-1} \in \mathcal{H}$ be given. Let $\mathcal{Z} \subset \mathcal{V}$ be a Banach space satisfying $\mathcal{Z} \subset L^{\infty}(\Omega)$ and $e^{\beta y} \in D(A) \cap \mathcal{Z}$ for all $y \in \mathcal{Z}$ and $\beta>0$. Defining $\delta_{j}=$ $\alpha_{j}-\alpha_{p} \beta_{j} / \beta_{p}$, we wish to solve the problem in the variable $y$,

$$
\frac{2}{\alpha \tau} e^{(1-\alpha / 2) y}\left(\frac{\alpha_{p}}{\beta_{p}} e^{\alpha y / 2}+\sum_{j=0}^{p-1} \delta_{j} v_{k+j}\right)+A\left(e^{y}\right)+\varepsilon L(y)=0 \quad \text { in } \mathcal{Z}^{\prime} .
$$

The relation to the original problem will be shown in (30) below. We have added a regularization operator $L: \mathcal{Z} \rightarrow \mathcal{Z}^{\prime}$, which is needed to derive uniform estimates in terms of $y$. The operator $L$ is supposed to satisfy the following conditions:

(iii) There exist $C>0$ and $\kappa_{1}>0$ such that $\left\langle L(y), e^{(\alpha-1) y}\right\rangle \geq-C$ and $\langle L(y), y\rangle \geq$ $\kappa_{1}\|y\|_{\mathcal{Z}}^{2}$ for all $y \in \mathcal{Z}$.

(iv) There exists a linear bounded operator $\widetilde{L}[z]: \mathcal{Z} \rightarrow \mathcal{Z}^{\prime}$ ("linearization" of $L$ around $z \in \mathcal{Z})$ such that $\widetilde{L}[y](y)=L(y)$ for all $y \in \mathcal{Z}$ and $\langle\widetilde{L}[z](y), y\rangle \geq \kappa_{0}\|y\|_{\mathcal{Z}}^{2}$ for all $e^{z} \in D(A)$ and $y \in \mathcal{Z}$, where $\kappa_{0}>0$.

The first condition in (iii) is important to derive the discrete entropy estimates; the second condition ensures the coercivity of a suitable bilinear form in the Lax-Milgram argument. Again, Assumption (iv) is employed in the "linearization" of the problem.

We claim that any solution $y \in \mathcal{Z}$ to (29) defines a solution to

$$
\frac{2}{\tau} \int_{\Omega} h\left(w_{k}\right)^{1 / 2} h^{\prime}\left(w_{k}\right)^{-1} \rho(E) v_{k} \phi d x+\left\langle A\left(w_{k}\right), \phi\right\rangle+\varepsilon\langle L(y), \phi\rangle=0
$$


for all $\phi \in \mathcal{Z}$, where $w_{k}=e^{y} \in D(A)$ and $h\left(w_{k}\right)^{1 / 2} h^{\prime}\left(w_{k}\right)^{-1}=(1 / \alpha) w_{k}^{1-\alpha / 2}$. Indeed, we define

$$
v_{k+p}:=\frac{1}{\beta_{p}} w_{k}^{\alpha / 2}-\sum_{j=0}^{p-1} \frac{\beta_{j}}{\beta_{p}} v_{k+j} \in \mathcal{H} .
$$

Then we insert $v_{k+p}$ in the definition (6) of $\sigma(E) v_{k}$, leading to

$$
\sigma(E) v_{k}=\sum_{k=0}^{p-1} \beta_{j} v_{k+j}+\beta_{p} v_{k+p}=w_{k}^{\alpha / 2} .
$$

Since $w_{k}=e^{y}>0$, we infer that $\sigma(E) v_{k}>0$. Inserting (31) in the definition (6) of $\rho(E) v_{k}$, we find that

$$
\begin{aligned}
\rho(E) v_{k} & =\alpha_{p} v_{k+p}+\sum_{j=0}^{p-1} \alpha_{j} v_{k+j}=\frac{\alpha_{p}}{\beta_{p}} w_{k}^{\alpha / 2}+\sum_{j=0}^{p-1}\left(\alpha_{j}-\frac{\alpha_{p}}{\beta_{p}} \beta_{j}\right) v_{k+j} \\
& =\frac{\alpha_{p}}{\beta_{p}} e^{\alpha y / 2}+\sum_{j=0}^{p-1} \delta_{j} v_{k+j},
\end{aligned}
$$

employing the definition $\delta_{j}=\alpha_{j}-\alpha_{p} \beta_{j} / \beta_{p}$. Replacing the brackets in (29) by $\rho(E) v_{k}$, we conclude that $\left(v_{k+p}, w_{k}\right)$ solves (30).

Step 2: Definition of the fixed-point operator. Let $\mathcal{X}$ be a Banach space such that the embedding $\mathcal{Z} \hookrightarrow \mathcal{X}$ is compact and $\mathcal{X} \subset L^{\infty}(\Omega)$. Let $z \in \mathcal{X}, \eta \in[0,1]$ be given. We define on $\mathcal{Z}$ the linear forms

$$
\begin{aligned}
a(y, \phi) & =\left\langle\widetilde{A}\left[e^{z}\right](y), \phi\right\rangle+\varepsilon\langle\widetilde{L}[z](y), \phi\rangle \\
F(\phi) & =-\frac{2 \eta}{\alpha \tau} \int_{\Omega} e^{(1-\alpha / 2) z}\left(\frac{\alpha_{p}}{\beta_{p}} e^{\alpha z / 2}+\sum_{j=0}^{p-1} \delta_{j} v_{k+j}\right) \phi d x .
\end{aligned}
$$

Note that $e^{\gamma z}$ is well defined for all $\gamma>0$ since $z \in \mathcal{Z} \subset L^{\infty}(\Omega)$. We wish to find $y \in \mathcal{Z}$ such that

$$
a(y, \phi)=F(\phi) \quad \text { for all } \phi \in \mathcal{Z} .
$$

By Assumptions (ii) and (iv), the forms $a$ and $F$ are continuous on $\mathcal{Z}$. The bilinear form $a$ is coercive since, by Assumptions (ii) and (iv),

$$
a(y, y)=\left\langle\widetilde{A}\left[e^{z}\right](y), y\right\rangle+\varepsilon\langle\widetilde{L}[z](y), \phi\rangle \geq \varepsilon \kappa_{0}\|y\|_{\mathcal{Z}}^{2}
$$

for all $y \in \mathcal{Z}$. The Lax-Milgram lemma provides a unique solution $y \in \mathcal{Z}$ to (33).

This defines the fixed-point operator $S: \mathcal{X} \times[0,1] \rightarrow \mathcal{X}, S(z, \eta)=y$. We have to show that $S$ is continuous and compact and that $S(z, 0)=0$ for all $z \in \mathcal{X}$. For these properties, more specific conditions on $A$ need to be imposed, and we refer to the following subsections. For instance, if $\widetilde{A}\left[e^{z}\right]+\varepsilon \widetilde{L}[z]$ is one-to-one, it follows that $S(z, 0)=0$, and the compactness is a consequence of the compactness of the embedding $\mathcal{Z} \hookrightarrow \mathcal{X}$. It remains 
to prove a uniform bound for all fixed points of $S(\cdot, \eta)$. A key ingredient is the following lemma which allows us to estimate the discrete time derivative.

Lemma 7 (Estimation of the discrete time derivative I). Let $1<\alpha<2$. The following estimate holds:

$$
\int_{\Omega}\left(\frac{\alpha_{p}}{\beta_{p}} e^{y}+e^{(1-\alpha / 2) y} \sum_{j=0}^{p-1} \delta_{j} v_{k+j}\right) y d x \geq-C\left(1+\sum_{j=0}^{p-1}\left\|v_{k+j}\right\|_{L^{2}(\Omega)}^{2}\right),
$$

and the constant $C>0$ only depends on $p, \alpha, \alpha_{j}$, and $\beta_{j}(j=0, \ldots, p)$.

Proof. We apply the Young inequality to the second summand of the integrand:

$$
\begin{aligned}
& \int_{\Omega}\left(\frac{\alpha_{p}}{\beta_{p}} y e^{y}+y e^{(1-\alpha / 2) y} \sum_{j=0}^{p-1} \delta_{j} v_{k+j}\right) d x \\
& \quad \geq \int_{\{y<0\}}\left(\frac{\alpha_{p}}{\beta_{p}} y e^{y}-\frac{1}{2} y^{2} e^{(2-\alpha) y}-\frac{1}{2}\left(\sum_{j=0}^{p-1} \delta_{j} v_{k+j}\right)^{2}\right) d x \geq-C-\frac{p}{2} \sum_{j=0}^{p-1} \delta_{j}^{2} \int_{\Omega} v_{k+j}^{2} d x .
\end{aligned}
$$

The last inequality follows from the fact that the mapping $x \mapsto\left(\alpha_{p} / \beta_{p}\right) x e^{x}-\frac{1}{2} x^{2} e^{(2-\alpha) x}$ for $x \in \mathbb{R}$ is bounded from below. For this statement, we need the condition $1<\alpha<2$.

Step 3: Uniform estimates. Let $y \in \mathcal{X}$ be a fixed point of $S(\cdot, \eta)$ and $\eta \in[0,1]$. By construction, $y \in \mathcal{Z}$ solves problem (29). With the test function $\phi=y$ in the weak formulation of (29), it follows, by Assumptions (i) and (iii) and by Lemma 7 , that

$$
\varepsilon \kappa_{1}\|y\|_{\mathcal{Z}}^{2} \leq\left\langle A\left(e^{y}\right), y\right\rangle+\varepsilon\langle L(y), y\rangle \leq \frac{C \eta}{\tau}\left(1+\sum_{j=0}^{p-1}\left\|v_{k+j}\right\|_{L^{2}(\Omega)}^{2}\right) .
$$

As a consequence, we obtain an $\varepsilon$-dependent bound which is uniform in $y \in \mathcal{Z}$ and $\eta \in[0,1]$ and, because of the continuous embedding $\mathcal{Z} \hookrightarrow \mathcal{X}$, also uniform in $y \in \mathcal{X}$. Thus, the fixedpoint theorem of Leray-Schauder provides the existence of a fixed point $y$ of $S(\cdot, 1)$, i.e., a solution to (29).

Step 4: Discrete entropy estimate. We derive estimates independent of $\varepsilon$. For this, we use the test function $\phi=w_{k}^{\alpha-1}:=e^{(\alpha-1) y} \in \mathcal{Z}$ in (29) (with $\eta=1$ ):

$$
\frac{2}{\alpha \tau} \int_{\Omega}\left(\frac{\alpha_{p}}{\beta_{p}} e^{y}+e^{(1-\alpha / 2) y} \sum_{j=0}^{p-1} \delta_{j} v_{k+j}\right) e^{(\alpha-1) y} d x+\left\langle A\left(e^{y}\right)+\varepsilon L(y), e^{(\alpha-1) y}\right\rangle=0 .
$$

The discrete time derivative is estimated as follows.

Lemma 8 (Estimation of the discrete time derivative II). The following estimate holds:

$$
\frac{2}{\alpha \tau} \int_{\Omega}\left(\frac{\alpha_{p}}{\beta_{p}} e^{y}+e^{(1-\alpha / 2) y} \sum_{j=0}^{p-1} \delta_{j} v_{k+j}\right) e^{(\alpha-1) y} d x \geq \frac{2}{\alpha \tau}\left(H\left[V_{k+1}\right]-H\left[V_{k}\right]\right) .
$$


Proof. Using the definition $\delta_{j}=\alpha_{j}-\alpha_{p} \beta_{j} / \beta_{p}$ and definition (31) for $v_{k+p}$, we can write the integrand as

$$
\begin{aligned}
\frac{\alpha_{p}}{\beta_{p}} e^{\alpha y}+e^{\alpha y / 2} \sum_{j=0}^{p-1} \delta_{j} v_{k+j} & =e^{\alpha y / 2}\left(\alpha_{p} v_{k+p}+\sum_{j=0}^{p-1} \alpha_{j} v_{k+j}\right) \\
& =w_{k}^{\alpha / 2} \rho(E) v_{k}=\sigma(E) v_{k} \rho(E) v_{k} .
\end{aligned}
$$

With the G-stability of the scheme $(\rho, \sigma)$, it follows from the proof of Proposition 5 that

$$
\begin{aligned}
\frac{2}{\alpha \tau} \int_{\Omega}\left(\frac{\alpha_{p}}{\beta_{p}} e^{\alpha y}+e^{\alpha y / 2} \sum_{j=0}^{p-1} \delta_{j} v_{k+j}\right) d x & =\frac{2}{\alpha \tau} \int_{\Omega} \sigma(E) v_{k} \rho(E) v_{k} d x \\
& \geq \frac{2}{\alpha \tau}\left(H\left[V_{k+1}\right]-H\left[V_{k}\right]\right),
\end{aligned}
$$

ending the proof.

By Assumption (iii), $\left\langle L(y), e^{(\alpha-1) y}\right\rangle \geq-C$. Therefore, using Lemma 8, (35) becomes

$$
H\left[V_{k+1}\right]+\frac{\alpha \tau}{2}\left\langle A\left(e^{y}\right), e^{(\alpha-1) y}\right\rangle \leq \frac{\varepsilon \alpha \tau C}{2}+H\left[V_{k}\right]
$$

This is the key inequality to derive the $\varepsilon$-independent bounds (observe that $\varepsilon<1$ ). Depending on the properties on the operator $A$, the expression $\left\langle A\left(e^{y}\right), e^{(\alpha-1) y}\right\rangle$ may yield certain Sobolev estimates (see Sections 4.2 and 4.3 for examples). Assumption (i) ensures that this term is at least nonnegative, and this is sufficient to derive $L^{\alpha}$ estimates for $w_{k}$.

Lemma 9. There exists a constant $C>0$ such that for all $k \in \mathbb{N}$,

$$
\left\|v_{k+p}\right\|_{L^{2}(\Omega)}+\left\|w_{k}^{\alpha / 2}\right\|_{L^{2}(\Omega)} \leq C,
$$

where $C>0$ does not depend on $\varepsilon$.

Proof. The positive definiteness of the matrix $G$ (with some constant $C_{G}>0$ ) implies that

$$
\begin{aligned}
H\left[V_{k+1}\right] & =\frac{1}{2} \sum_{j=0}^{p-1} G_{i j} \int_{\Omega} v_{k+1+i} v_{k+1+j} d x \geq \frac{C_{G}}{2} \sum_{j=0}^{p-1} \int_{\Omega} v_{k+1+j}^{2} d x \\
& =\frac{C_{G}}{2} \int_{\Omega} v_{k+p}^{2} d x+\frac{C_{G}}{2} \sum_{j=1}^{p-1} \int_{\Omega} v_{k+j}^{2} d x .
\end{aligned}
$$

In view of (36), this provides a uniform estimate for $v_{k+p}$ in $L^{2}\left(\mathbb{T}^{d}\right)$. Furthermore,

$$
\int_{\Omega} w_{k}^{\alpha} d x=\int_{\Omega}\left(\sigma(E) v_{k}\right)^{2} d x \leq(p+1) \int_{\Omega} \sum_{j=0}^{p} \beta_{j}^{2} v_{k+j}^{2} d x,
$$

and we conclude a uniform estimate for $w_{k}$ in $L^{\alpha}(\Omega)$. 
Step 5: Limit $\varepsilon \rightarrow 0$ in (29). Set $w_{\varepsilon}=w_{k}=e^{y}, v_{\varepsilon}=v_{k+p}$, and $y_{\varepsilon}=y$. Then, using Lemma 9 and (34),

$$
\left\|v_{\varepsilon}\right\|_{L^{2}(\Omega)}+\left\|w_{\varepsilon}^{\alpha / 2}\right\|_{L^{2}(\Omega)}+\sqrt{\varepsilon}\left\|y_{\varepsilon}\right\|_{\mathcal{Z}} \leq C .
$$

The limit $\varepsilon \rightarrow 0$ depends on the specific structure of $A$ and cannot be detailed here without further assumptions. We refer to the examples presented below. If we are able to prove that $v_{\varepsilon} \rightarrow v, w_{\varepsilon} \rightarrow w$ in appropriate spaces for some $v, w$ and if the limit functions satisfy (28), we can set $v_{k+p}:=v$ and $w_{k}:=w$.

Step 6: Discrete entropy dissipation. The weak convergence $v_{\varepsilon} \rightarrow v_{k+p}$ in $L^{2}(\Omega)$ for a subsequence (see (37)) and the lower semi-continuity of $u \mapsto\|u\|_{L^{2}(\Omega)}^{2}$ on $L^{2}(\Omega)$ yield

$$
\begin{aligned}
& \liminf _{\varepsilon \rightarrow 0} H\left[v_{k+1}, \ldots, v_{k+p-1}, v_{\varepsilon}\right]=\sum_{i, j=0}^{p-2} G_{i j} \int_{\Omega} v_{k+1+i} v_{k+1+j} d x \\
& \quad+2 \lim _{\varepsilon \rightarrow 0} \sum_{j=0}^{p-2} G_{p-1, j} \int_{\Omega} v_{\varepsilon} v_{k+1+j} d x+\liminf _{\varepsilon \rightarrow 0} G_{p-1, p-1} \int_{\Omega} v_{\varepsilon}^{2} d x \\
& \geq \sum_{i, j=0}^{p-2} G_{i j} \int_{\Omega} v_{k+1+i} v_{k+1+j} d x+2 \sum_{j=0}^{p-2} G_{p-1, j} \int_{\Omega} v_{k+p} v_{k+1+j} d x+G_{p-1, p-1} \int_{\Omega} v_{k+p}^{2} d x \\
& =H\left[v_{k+1}, \ldots, v_{k+p-1}, v_{k+p}\right]=H\left[V_{k+1}\right] .
\end{aligned}
$$

Assuming that

$$
\liminf _{\varepsilon \rightarrow 0}\left\langle A\left(e^{y_{\varepsilon}}\right), e^{(\alpha-1) y_{\varepsilon}}\right\rangle \geq\left\langle A\left(e^{y}\right), e^{(\alpha-1) y}\right\rangle,
$$

the limit $\varepsilon \rightarrow 0$ in (36) shows that

$$
H\left[V_{k+1}\right]+\frac{\alpha \tau}{2}\left\langle A\left(e^{y}\right), e^{(\alpha-1) y}\right\rangle \leq H\left[V_{k}\right],
$$

which is the desired discrete entropy dissipation inequality.

4.2. A cross-diffusion population system. We make the arguments of the previous subsection rigorous for the cross-diffusion system (11)-(13). Let $d \leq 3$ and define the spaces $\mathcal{H}=L^{2}\left(\mathbb{T}^{d}\right)^{2}, \mathcal{V}=H^{1}\left(\mathbb{T}^{d}\right)^{2}, \mathcal{Z}=H^{2}\left(\mathbb{T}^{d}\right)^{2}, \mathcal{X}=W^{1,4}\left(\mathbb{T}^{d}\right)^{2}$, and $D(A)=\{u=$ $\left.\left(u^{(1)}, u^{(2)}\right) \in W^{1,4}\left(\mathbb{T}^{d}\right)^{2}: u^{(1)}>0, u^{(2)}>0\right\}$. The operator $A: D(A) \rightarrow \mathcal{V}^{\prime}$ is given by

$$
\begin{aligned}
\langle A(u), \phi\rangle= & \int_{\mathbb{T}^{d}}\left(\left(d_{1}+a_{1} u^{(1)}+u^{(2)}\right) \nabla u^{(1)} \cdot \nabla \phi^{(1)}+u^{(1)} \nabla u^{(2)} \cdot \nabla \phi^{(1)}\right. \\
& \left.+\left(d_{2}+a_{2} u^{(2)}+u^{(1)}\right) \nabla u^{(2)} \cdot \nabla \phi^{(2)}+u^{(2)} \nabla u^{(1)} \cdot \nabla \phi^{(2)}\right) d x
\end{aligned}
$$

for $u=\left(u^{(1)}, u^{(2)}\right) \in D(A)$ and $\phi=\left(\phi^{(1)}, \phi^{(2)}\right) \in \mathcal{V}$. We have to verify Assumptions (i)(iv) stated in Section 4.1. We show first that (i) is satisfied. In the following, we define $f(u)=\left(f\left(u^{(1)}\right), f\left(u^{(2)}\right)\right)$ for arbitrary functions $f: \mathbb{R}^{2} \rightarrow \mathbb{R}$ and $u=\left(u^{(1)}, u^{(2)}\right)$. 
Lemma 10. Let $1<\alpha \leq 2, u \in D(A)$, and $4 a_{1} a_{2} \geq \max \left\{a_{1}, a_{2}\right\}+1$. Then

$$
\begin{aligned}
& \langle A(u), \log u\rangle \geq 4 \int_{\mathbb{T}^{d}}\left(d_{1}\left|\nabla \sqrt{u^{(1)}}\right|^{2}+d_{2}\left|\nabla \sqrt{u^{(2)}}\right|^{2}\right) d x, \\
& \left\langle A(u), u^{\alpha-1}\right\rangle \geq \frac{4(\alpha-1)}{\alpha^{2}} \int_{\mathbb{T}^{d}}\left(d_{1}\left|\nabla\left(u^{(1)}\right)^{\alpha / 2}\right|^{2}+d_{2}\left|\nabla\left(u^{(2)}\right)^{\alpha / 2}\right|^{2}\right) d x .
\end{aligned}
$$

Proof. The first inequality follows from

$$
\begin{aligned}
\langle A(u), \log u\rangle= & \int_{\mathbb{T}^{d}}\left(d_{1} \frac{\left|\nabla u^{(1)}\right|^{2}}{u^{(1)}}+d_{2} \frac{\left|\nabla u^{(2)}\right|^{2}}{u^{(2)}}+a_{1}\left|\nabla u^{(1)}\right|^{2}+a_{2}\left|\nabla u^{(2)}\right|^{2}\right. \\
& \left.+4\left|\nabla \sqrt{u^{(1)} u^{(2)}}\right|^{2}\right) d x .
\end{aligned}
$$

To prove the second inequality, we calculate

$$
\begin{aligned}
\left\langle A(u), u^{\alpha-1}\right\rangle= & (\alpha-1) \int_{\mathbb{T}^{d}}\left(\left(d_{1}+a_{1} u^{(1)}+u^{(2)}\right)\left(u^{(1)}\right)^{\alpha-2}\left|\nabla u^{(1)}\right|^{2}+\left(u^{(1)}\right)^{\alpha-1} \nabla u^{(2)} \cdot \nabla u^{(1)}\right. \\
& \left.+\left(d_{2}+a_{2} u^{(2)}+u^{(1)}\right)\left(u^{(2)}\right)^{\alpha-2}\left|\nabla u^{(2)}\right|^{2}+\left(u^{(2)}\right)^{\alpha-1} \nabla u^{(1)} \cdot \nabla u^{(2)}\right) d x .
\end{aligned}
$$

For $u^{(1)} \leq u^{(2)}$, we find that

$$
\begin{aligned}
\left(\left(u^{(1)}\right)^{\alpha-1}+\right. & \left.\left(u^{(2)}\right)^{\alpha-1}\right) \nabla u^{(1)} \cdot \nabla u^{(2)} \geq-a_{1}\left(u^{(1)}\right)^{\alpha-1}\left|\nabla u^{(1)}\right|^{2} \\
& -\frac{1}{4 a_{1}}\left(u^{(1)}\right)^{\alpha-1}\left|\nabla u^{(2)}\right|^{2}-\frac{1}{4}\left(u^{(2)}\right)^{\alpha-1}\left|\nabla u^{(2)}\right|^{2}-u^{(2)}\left(u^{(2)}\right)^{\alpha-2}\left|\nabla u^{(1)}\right|^{2} \\
\geq & -a_{1}\left(u^{(1)}\right)^{\alpha-1}\left|\nabla u^{(1)}\right|^{2}-\left(\frac{1}{4}+\frac{1}{4 a_{1}}\right)\left(u^{(2)}\right)^{\alpha-1}\left|\nabla u^{(2)}\right|^{2}-u^{(2)}\left(u^{(1)}\right)^{\alpha-2}\left|\nabla u^{(1)}\right|^{2} .
\end{aligned}
$$

Here, we have used the inequalities $\left(u^{(1)}\right)^{\alpha-1} \leq\left(u^{(2)}\right)^{\alpha-1}$ and $\left(u^{(2)}\right)^{\alpha-2} \leq\left(u^{(1)}\right)^{\alpha-2}$, noting that $1<\alpha \leq 2$. In a similar way, it follows for $u^{(1)}>u^{(2)}$ that

$$
\begin{aligned}
\left(\left(u^{(1)}\right)^{\alpha-1}+\right. & \left.\left(u^{(2)}\right)^{\alpha-1}\right) \nabla u^{(1)} \cdot \nabla u^{(2)} \geq-a_{2}\left(u^{(2)}\right)^{\alpha-1}\left|\nabla u^{(2)}\right|^{2} \\
& -\left(\frac{1}{4}+\frac{1}{4 a_{2}}\right)\left(u^{(1)}\right)^{\alpha-1}\left|\nabla u^{(1)}\right|^{2}-u^{(1)}\left(u^{(2)}\right)^{\alpha-2}\left|\nabla u^{(2)}\right|^{2} .
\end{aligned}
$$

This shows that

$$
\begin{aligned}
\left\langle A(u), u^{\alpha-1}\right\rangle \geq & \frac{4(\alpha-1)}{\alpha^{2}} \int_{\mathbb{T}^{d}}\left(d_{1}\left|\nabla\left(u^{(1)}\right)^{\alpha / 2}\right|^{2}+d_{2}\left|\nabla\left(u^{(2)}\right)^{\alpha / 2}\right|^{2}\right) d x \\
& +\frac{\alpha-1}{4 a_{2}}\left(4 a_{1} a_{2}-a_{2}-1\right) \int_{\mathbb{T}^{2}}\left(u^{(1)}\right)^{\alpha-1}\left|\nabla u^{(1)}\right|^{2} d x \\
& +\frac{\alpha-1}{4 a_{1}}\left(4 a_{1} a_{2}-a_{1}-1\right) \int_{\mathbb{T}^{2}}\left(u^{(2)}\right)^{\alpha-1}\left|\nabla u^{(2)}\right|^{2} d x \geq 0
\end{aligned}
$$

since the assumption on $a_{1}, a_{2}$ implies that $4 a_{1} a_{2}-a_{2}-1 \geq 0$ and $4 a_{1} a_{2}-a_{1}-1 \geq 0$. 
The regularization $L: \mathcal{Z} \rightarrow \mathcal{Z}^{\prime}$ is defined by

$$
\langle L(y), \phi\rangle=\sum_{m=1}^{2} \int_{\mathbb{T}^{d}}\left(\Delta y^{(m)} \Delta \phi^{(m)}+\left|\nabla y^{(m)}\right|^{2} \nabla y^{(m)} \cdot \nabla \phi^{(m)}+y^{(m)} \phi^{(m)}\right) d x
$$

for $y=\left(y^{(1)}, y^{(2)}\right), \phi=\left(\phi^{(1)}, \phi^{(2)}\right) \in \mathcal{Z}$. We show that it fulfills Assumption (iii).

Lemma 11. It holds for all $y \in \mathcal{Z}$

$$
\left\langle L(y), e^{(\alpha-1) y}\right\rangle \geq-\frac{1}{e(\alpha-1)}, \quad\langle L(y), y\rangle \geq \kappa_{1}\|y\|_{\mathcal{Z}}^{2},
$$

where $\kappa_{1}>0$ only depends on the Poincaré constant for periodic functions with vanishing integral mean.

Proof. A a straightforward computation shows that for $m=1,2$,

$$
\begin{aligned}
\Delta y^{(m)} & \Delta\left(e^{(\alpha-1) y^{(m)}}\right)+\left|\nabla y^{(m)}\right|^{2} \nabla y^{(m)} \cdot \nabla\left(e^{(\alpha-1) y^{(m)}}\right) \\
= & 4(\alpha-1) e^{(\alpha-1) y^{(m)}}\left(\frac{\Delta e^{y^{(m)} / 2}}{e^{y^{(m)} / 2}}-(2-\alpha)\left|\frac{\nabla e^{y^{(m)} / 2}}{e^{y^{(m)} / 2}}\right|^{2}\right)^{2} \\
& +4\left(\alpha^{2}-1\right)(3-\alpha)\left|\frac{\nabla e^{y^{(m)} / 2}}{e^{y^{(m)} / 2}}\right|^{4} \geq 0 .
\end{aligned}
$$

Then the first inequality follows from the fact that the mapping $x \mapsto x e^{(\alpha-1) x}, x \in \mathbb{R}$, is bounded from below by $-1 /(e(\alpha-1))$. The second inequality is a consequence of

$$
\begin{aligned}
\|y\|_{H^{2}\left(\mathbb{T}^{2}\right)^{2}}^{2} & =\sum_{m=1}^{2} \int_{\mathbb{T}^{d}}\left(\left\|\nabla^{2} y^{(m)}\right\|^{2}+\left|\nabla y^{(m)}\right|^{2}+\left(y^{(m)}\right)^{2}\right) d x \\
& \leq \sum_{m=1}^{2} \int_{\mathbb{T}^{d}}\left(\left(C^{2}+1\right)\left\|\nabla^{2} y^{(m)}\right\|^{2}+\left(y^{(m)}\right)^{2}\right) d x \\
& \leq\left(C^{2}+1\right) \sum_{m=1}^{2} \int_{\mathbb{T}^{d}}\left(\left(\Delta y^{(m)}\right)^{2}+\left(y^{(m)}\right)^{2}\right) d x \leq\left(C^{2}+1\right)\langle L(y), y\rangle,
\end{aligned}
$$

where $C>0$ is the Poincaré constant.

The "linearization" of $A$ is defined by "freezing" the diffusion coefficients:

$$
\begin{aligned}
\left\langle\widetilde{A}\left[e^{z}\right](y), \phi\right\rangle= & \int_{\mathbb{T}^{d}}\left(\left(d_{1}+a_{1} e^{z^{(1)}}+e^{z^{(2)}}\right) e^{z^{(1)}} \nabla y^{(1)}+e^{z^{(1)}+z^{(2)}} \nabla y^{(2)}\right) \cdot \nabla \phi^{(1)} d x \\
& +\int_{\mathbb{T}^{d}}\left(\left(d_{2}+a_{2} e^{z^{(2)}}+e^{z^{(1)}}\right) e^{z^{(2)}} \nabla y^{(2)}+e^{z^{(1)}+z^{(2)}} \nabla y^{(1)}\right) \cdot \nabla \phi^{(2)} d x,
\end{aligned}
$$

for $z=\left(z^{(1)}, z^{(2)}\right) \in \mathcal{X}=W^{1,4}\left(\mathbb{T}^{d}\right)^{2}, y, \phi \in \mathcal{V}$. This operator satisfies Assumption (ii):

$$
\left\langle\widetilde{A}\left[e^{z}\right](y), y\right\rangle=\int_{\mathbb{T}^{d}}\left(\left(d_{1}+a_{1} e^{2 z^{(1)}}\right)\left|\nabla y^{(1)}\right|^{2}+\left(d_{2}+a_{2} e^{2 z^{(2)}}\right)\left|\nabla y^{(12)}\right|^{2}\right.
$$




$$
\left.+e^{z^{(1)}+z^{(2)}}\left|\nabla\left(y^{(1)}+y^{(2)}\right)\right|^{2}\right) d x \geq 0, \quad y \in \mathcal{Z},
$$

Similarly, we define $\widetilde{L}[z]$ for $z \in \mathcal{X}$ and $y, \phi \in \mathcal{Z}$ by

$$
\langle\widetilde{L}[z](y), \phi\rangle=\sum_{m=1}^{2} \int_{\mathbb{T}^{d}}\left(\Delta y^{(m)} \Delta \phi^{(m)}+\left|\nabla z^{(m)}\right|^{2} \nabla y^{(m)} \cdot \nabla \phi^{(m)}+y^{(m)} \phi^{(m)}\right) d x,
$$

fulfilling Assumption (iv).

In Step 2 in Section 4.1, we have defined a fixed-point operator $S: \mathcal{X} \times[0,1] \rightarrow \mathcal{X}$. It is not difficult to show that this operator is continuous and compact, taking into account the compactness of the embedding $\mathcal{Z}=H^{2}\left(\mathbb{T}^{d}\right)^{2} \hookrightarrow \mathcal{X}=W^{1,4}\left(\mathbb{T}^{d}\right)^{2}$. In order to show that $S(z, 0)=0$ for $z \in \mathcal{Z}$, we write

$$
\widetilde{A}\left[e^{z}\right](y)=-\operatorname{div}(D(z) \nabla y),
$$

where the diffusion matrix

$$
D(z)=\left(\begin{array}{cc}
\left(d_{1}+a_{1} e^{z^{(1)}}+e^{z^{(2)}}\right) e^{z^{(1)}} & e^{z^{(1)}+z^{(2)}} \\
e^{z^{(1)}+z^{(2)}} & \left(d_{2}+a_{2} e^{z^{(2)}}+e^{z^{(1)}}\right) e^{z^{(2)}}
\end{array}\right),
$$

is symmetric and positive definite. Therefore, $\widetilde{A}\left[e^{z}\right](y)+\varepsilon \widetilde{L}[z](y)$ is one-to-one, showing that $S(z, 0)=0$. We infer from Steps 1-3 in Section 4.1 that there exists a weak solution $y \in \mathcal{Z}$ to (29). It remains to derive discrete entropy estimates (independent of $\varepsilon$ ) and to perform the limit $\varepsilon \rightarrow 0$. Employing the test function $\phi=w_{k}^{\alpha-1}=e^{(\alpha-1) y} \in \mathcal{Z}$ in the weak formulation of (29) and taking into account Lemmas 8, 10, and 11, we obtain the discrete entropy estimate

$$
H\left[V_{k+1}\right]+\frac{2 \tau}{\alpha}(\alpha-1) \int_{\mathbb{T}^{d}}\left(d_{1}\left|\nabla\left(w_{k}^{(1)}\right)^{\alpha / 2}\right|^{2}+d_{2}\left|\nabla\left(w_{k}^{(1)}\right)^{\alpha / 2}\right|^{2}\right) d x \leq \frac{\varepsilon \alpha \tau}{2 e(\alpha-1)}+H\left[V_{k}\right]
$$

This estimate and (40) are sufficient to perform the limit $\varepsilon \rightarrow 0$. Set $w_{\varepsilon}=w_{k}=e^{y}$, $v_{\varepsilon}=v_{k+p}$ (defined in (31) $)$, and $y_{\varepsilon}=y$. Because of Lemma 9 and (41), we have the $\varepsilon$-independent bounds

$$
\left\|v_{\varepsilon}^{(j)}\right\|_{L^{2}\left(\mathbb{T}^{d}\right)}+\left\|\left(w_{\varepsilon}^{(j)}\right)^{\alpha / 2}\right\|_{H^{1}\left(\mathbb{T}^{d}\right)}+\sqrt{\varepsilon}\left\|y_{\varepsilon}^{(j)}\right\|_{H^{2}\left(\mathbb{T}^{d}\right)} \leq C, \quad j=1,2 .
$$

By compactness, there exist subsequences, which are not relabeled, such that, as $\varepsilon \rightarrow 0$, for $j=1,2$,

$$
\begin{aligned}
v_{\varepsilon}^{(j)} \rightarrow v^{(j)} & \text { weakly in } L^{2}\left(\mathbb{T}^{d}\right), \\
\left(w_{\varepsilon}^{(j)}\right)^{\alpha / 2} \rightarrow z^{(j)} & \text { weakly in } H^{1}\left(\mathbb{T}^{d}\right), \\
\left(w_{\varepsilon}^{(j)}\right)^{\alpha / 2} \rightarrow z^{(j)} & \text { strongly in } L^{6}\left(\mathbb{T}^{d}\right), \\
\varepsilon y_{\varepsilon}^{(j)} \rightarrow 0 & \text { strongly in } H^{2}\left(\mathbb{T}^{d}\right) \text { and in } W^{1,4}\left(\mathbb{T}^{d}\right) .
\end{aligned}
$$

The last limit implies that $\varepsilon L\left(y_{\varepsilon}\right) \rightarrow 0$ in $H^{-2}\left(\mathbb{T}^{d}\right)^{2}$.

In view of $0<\exp \left(\alpha y_{\varepsilon}^{(j)} / 2\right)=\left(w_{\varepsilon}^{(j)}\right)^{\alpha / 2}=\sigma(E) v_{\varepsilon}^{(j)}$ and the linearity of the operator $\sigma(E)$, it follows that $0 \leq z^{(j)}=\sigma(E) v^{(j)}$, where $\sigma(E) v^{(j)}=\beta_{p} v^{(j)}+\sum_{m=0}^{p-1} \beta_{m} v_{k+m}^{(j)}$. This 
allows us to define $w:=z^{2 / \alpha}$, where $z=\left(z^{(1)}, z^{(2)}\right)$. Since $1<\alpha<2, w_{\varepsilon}^{1-\alpha / 2} \rightarrow w^{1-\alpha / 2}$ strongly in $L^{6}\left(\mathbb{T}^{d}\right)^{2}$. We infer that

$$
\begin{aligned}
\frac{2}{\alpha \tau}\left(\frac{\alpha_{p}}{\beta_{p}} e^{y_{\varepsilon}}+e^{(1-\alpha / 2) y_{\varepsilon}} \sum_{m=0}^{p-1} \delta_{m} v_{k+m}\right) & =\frac{2}{\alpha \tau} e^{(1-\alpha / 2) y_{\varepsilon}}\left(\frac{\alpha_{p}}{\beta_{p}} e^{\alpha y_{\varepsilon} / 2}+\sum_{m=0}^{p-1} \delta_{m} v_{k+m}\right) \\
& =\frac{2}{\alpha \tau} w_{\varepsilon}^{1-\alpha / 2} \rho(E) v_{\varepsilon} \\
& \rightarrow \frac{2}{\alpha \tau} w^{1-\alpha / 2} \rho(E) v \quad \text { weakly in } L^{3 / 2}\left(\mathbb{T}^{d}\right),
\end{aligned}
$$

where $\rho(E) v=\alpha_{p} v+\sum_{m=0}^{p-1} \alpha_{m} v_{k+m}$.

It remains to perform the limit $\varepsilon \rightarrow 0$ in the term involving $A$. We find that, for $j=1,2$,

$$
\nabla w_{\varepsilon}^{(j)}=\frac{2}{\alpha}\left(w_{\varepsilon}^{(j)}\right)^{1-\alpha / 2} \nabla\left(w_{\varepsilon}^{(j)}\right)^{\alpha / 2} \rightarrow \frac{2}{\alpha}\left(w^{(j)}\right)^{1-\alpha / 2} \nabla\left(w^{(j)}\right)^{\alpha / 2} \quad \text { weakly in } L^{3 / 2}\left(\mathbb{T}^{d}\right) .
$$

Since $\nabla w_{\varepsilon}^{(j)} \rightarrow \nabla w^{(j)}$ in the sense of distributions, (42) shows that $\nabla w_{\varepsilon}^{(j)} \rightarrow \nabla w^{(j)}$ weakly in $L^{3 / 2}\left(\mathbb{T}^{d}\right)$. We conclude that

$$
\begin{aligned}
\left\langle A\left(w_{\varepsilon}\right), \phi\right\rangle \rightarrow & \frac{2}{\alpha} \int_{\mathbb{T}^{d}}\left(\left(d_{1}+a_{1} w^{(1)}+w^{(2)}\right) \nabla w^{(1)} \cdot \nabla \phi^{(1)}+w^{(1)} \nabla w^{(2)} \cdot \nabla \phi^{(1)}\right. \\
& \left.+\left(d_{2}+a_{2} w^{(2)}+w^{(1)}\right) \nabla w^{(2)} \cdot \nabla \phi^{(2)}+w^{(2)} \nabla w^{(1)} \cdot \nabla \phi^{(2)}\right) d x
\end{aligned}
$$

for all $\phi \in W^{1, \infty}\left(\mathbb{T}^{d}\right)^{2}$. The limit $\varepsilon \rightarrow 0$ in (29) then yields (15)-(16). Finally, applying the limes inferior to (41) and using the weak convergence (42) and the lower semi-continuity of $u \mapsto\|\nabla u\|_{L^{2}\left(\mathbb{T}^{d}\right)}^{2}$ on $H^{1}\left(\mathbb{T}^{d}\right)$, inequality (17) follows. Setting $v_{k+p}:=v$ and $w_{k+p}:=w$, this finishes the proof of Theorem 1 .

4.3. The DLSS equation. We apply the general scheme (28) to the DLSS equation (18). For this, let $d \leq 3$ and define $\mathcal{H}=L^{2}\left(\mathbb{T}^{d}\right), \mathcal{V}=\mathcal{Z}=H^{2}\left(\mathbb{T}^{d}\right), \mathcal{X}=W^{1,4}\left(\mathbb{T}^{d}\right)$, $D(A)=\left\{u \in H^{2}\left(\mathbb{T}^{d}\right): u>0\right.$ in $\left.\mathbb{T}^{d}\right\}$, and $A: D(A) \rightarrow \mathcal{V}^{\prime}$ by

$$
\langle A(u), \phi\rangle=\left\langle\nabla^{2}:\left(u \nabla^{2} \log u\right), \phi\right\rangle=\int_{\mathbb{T}^{d}}\left(\nabla^{2} u-4 \nabla \sqrt{u} \otimes \nabla \sqrt{u}\right): \nabla^{2} \phi d x,
$$

for $u \in D(A), \phi \in \mathcal{V}$, where the tensor product $\nabla \sqrt{u} \otimes \nabla \sqrt{u}$ consists of the components $\left(\partial \sqrt{u} / \partial x_{i}\right)\left(\partial \sqrt{u} / \partial x_{j}\right)$. Observe that $u \in \mathcal{V}$ implies that $\sqrt{u} \in W^{1,4}\left(\mathbb{T}^{d}\right)$ by the LionsVillani lemma (see the version in [5, Lemma 26]) such that the integral on the right-hand side of the definition of $A$ is well defined. We need to verify Assumptions (i)-(iv) of Section 4.1 .

By Lemma 2.2 of [31], it holds that

$$
\begin{aligned}
& \langle A(u), \log u\rangle \geq \kappa_{0} \int_{\mathbb{T}^{d}}\left(\Delta u^{1 / 2}\right)^{2} d x, \\
& \left\langle A(u), u^{\alpha-1}\right\rangle \geq \kappa_{\alpha} \int_{\mathbb{T}^{d}}\left(\Delta u^{\alpha / 2}\right)^{2} d x
\end{aligned}
$$


for all $1<\alpha<2(d+1) /(d+2)$ and $u \in D(A)$, where $\kappa_{\alpha}>0$ for $\alpha \geq 1$ depends only on $\alpha$ and the space dimension $d$. This shows Assumption (i). The "linearization" of $A$ is defined by $\widetilde{A}[v](u)=\nabla^{2}:\left(v \nabla^{2} \log u\right)$ for $u, v \in D(A)$. The regularization $L$ and its "linearization" are defined similarly as in Section 4.2 .

$$
L(y)=\Delta^{2} y-\operatorname{div}\left(|\nabla y|^{2} \nabla y\right)+y, \quad \widetilde{L}[z](y)=\Delta^{2} y-\operatorname{div}\left(|\nabla z|^{2} \nabla y\right)+y
$$

for $y, z \in \mathcal{V}$ with $u=e^{y}, v=e^{z}$. By Lemma 11, Assumption (iii) is satisfied. Moreover, Assumptions (ii) and (iv) hold as well.

From Steps 1-3 of Section 4.1, we infer that there exists a weak solution $y \in \mathcal{V}$ to (29). The discrete entropy estimate follows from Lemmas 8 and 11 and estimate (43):

$$
H\left[V_{k+1}\right]+\frac{\alpha \tau}{2} \kappa_{\alpha} \int_{\mathbb{T}^{d}}\left(\Delta w_{k}^{\alpha / 2}\right)^{2} d x \leq \frac{\varepsilon \tau}{2 e(\alpha-1)}+H\left[V_{k}\right] .
$$

Together with the $L^{2}$-bound for $w_{k}^{\alpha / 2}$ from Lemma 9, we obtain the following $\varepsilon$-independent estimates

$$
\left\|v_{\varepsilon}\right\|_{L^{2}\left(\mathbb{T}^{d}\right)}+\left\|w_{\varepsilon}^{\alpha / 2}\right\|_{H^{2}\left(\mathbb{T}^{d}\right)}+\sqrt{\varepsilon}\left\|y_{\varepsilon}\right\|_{H^{2}\left(\mathbb{T}^{d}\right)} \leq C
$$

where $v_{\varepsilon}:=v_{k+p}$ (defined in (31) $), w_{\varepsilon}:=w_{k}$, and $y_{\varepsilon}:=y$. Arguing as in Section 4.2, there exist subsequences such that, as $\varepsilon \rightarrow 0$,

$$
\begin{aligned}
v_{\varepsilon} \rightarrow v & \text { weakly in } L^{2}\left(\mathbb{T}^{d}\right), \\
w_{\varepsilon}^{\alpha / 2} \rightarrow w^{\alpha / 2} & \text { weakly in } H^{2}\left(\mathbb{T}^{d}\right), \\
w_{\varepsilon}^{\alpha / 2} \rightarrow w^{\alpha / 2} & \text { strongly in } L^{\infty}\left(\mathbb{T}^{d}\right), \\
\varepsilon y_{\varepsilon} \rightarrow 0 & \text { strongly in } H^{2}\left(\mathbb{T}^{d}\right),
\end{aligned}
$$

where $w^{\alpha / 2}=\sigma(E) v$, and $\varepsilon L\left(y_{\varepsilon}\right) \rightarrow 0$ in $H^{-2}\left(\mathbb{T}^{d}\right)$.

We perform the limit $\varepsilon \rightarrow 0$ in the fourth-order operator. By the Lions-Villani lemma [5. Lemma 26],

Hence, because of

$$
\left\|w_{\varepsilon}^{\alpha / 4}\right\|_{W^{1,4}\left(\mathbb{T}^{d}\right)} \leq C\left\|w_{\varepsilon}^{\alpha / 2}\right\|_{H^{2}\left(\mathbb{T}^{d}\right)} \leq C
$$

$$
\left(w_{\varepsilon}^{\alpha / 4}\right) \text { is bounded in } W^{1,4}\left(\mathbb{T}^{d}\right), \quad w_{\varepsilon}^{\alpha / 2} \rightarrow w^{\alpha / 2} \text { strongly in } H^{1}\left(\mathbb{T}^{d}\right),
$$

and $\alpha / 4<1 / 2<\alpha / 2$, Proposition A.1 of [32] shows that

$$
\left|\nabla w_{\varepsilon}^{1 / 2}\right| \rightarrow\left|\nabla w^{1 / 2}\right| \quad \text { strongly in } L^{2 \alpha}\left(\mathbb{T}^{d}\right)
$$

Then the limit $\varepsilon \rightarrow 0$ gives

$$
\begin{aligned}
\nabla^{2}:\left(e^{y_{\varepsilon}} \nabla^{2} y_{\varepsilon}\right) & =\nabla^{2}:\left(\frac{2}{\alpha} w_{\varepsilon}^{1-\alpha / 2} \nabla^{2} w_{\varepsilon}^{\alpha / 2}-2 \alpha \nabla w_{\varepsilon}^{1 / 2} \otimes \nabla w_{\varepsilon}^{1 / 2}\right) \\
& \rightarrow \nabla^{2}:\left(\frac{2}{\alpha} w^{1-\alpha / 2} \nabla^{2} w^{\alpha / 2}-2 \alpha \nabla w^{1 / 2} \otimes \nabla w^{1 / 2}\right)
\end{aligned}
$$

weakly in $W^{-2, \alpha}\left(\mathbb{T}^{d}\right)$. Therefore, passing to the limit $\varepsilon \rightarrow 0$ in (29) yields (19)). Finally, the discrete entropy dissipation inequality (20) follows as at the end of the previous subsection 
from the weak convergence of $w_{\varepsilon}^{\alpha / 2}$ to $w^{\alpha / 2}$ in $H^{2}\left(\mathbb{T}^{d}\right)$ and the lower semi-continuity of $u \mapsto\|\Delta u\|_{L^{2}\left(\mathbb{T}^{d}\right)}^{2}$ on $H^{2}\left(\mathbb{T}^{d}\right)$.

\section{Convergence Rate}

In this section, we prove Theorem [3. Let $0<\tau<1$. The idea of the proof is to estimate the difference $\widehat{v}(t)=v(t)-\delta_{I}(t)$ (see (22) and [25, Theorem V.6.10]). Setting $B(v)=\frac{\alpha}{2} v^{1-2 / \alpha} A\left(v^{2 / \alpha}\right)$, we rewrite equation (7) for the exact solution as

$$
\tau v_{t}\left(t_{k+2}\right)+\tau B\left(v\left(t_{k+2}\right)\right)=0 .
$$

With the definitions (22) of $\delta_{D}$ and $\delta_{I}$, this can be formulated as

$$
\rho(E) \widehat{v}\left(t_{k}\right)+\tau B\left(\sigma(E) \widehat{v}\left(t_{k}\right)-\widehat{\varepsilon}\left(t_{k}\right)\right)=-\widehat{\delta}\left(t_{k}\right),
$$

where $\widehat{\delta}(t)=\delta_{D}(t)-\rho(E) \delta_{I}(t)$ and $\widehat{\varepsilon}(t)=\delta_{I}(t)-\sigma(E) \delta_{I}(t)$.

We derive bounds for $\widehat{\delta}$ and $\widehat{\varepsilon}$. A Taylor expansion of $\delta_{I}$ at $t=t_{k+1}$ and $t=t_{k+2}$ around $t_{k}$ and the condition $\sigma(1)=1$ yields

$$
\begin{aligned}
\sigma(E) \delta_{I}\left(t_{k}\right)= & \beta_{0} \delta_{I}\left(t_{k}\right)+\beta_{1}\left(\delta_{I}\left(t_{k}\right)+\tau \delta_{I}^{\prime}\left(t_{k}\right)+\frac{1}{2} \tau^{2} \delta_{I}^{\prime \prime}\left(t_{k}\right)\right) \\
& +\beta_{2}\left(\delta_{I}\left(t_{k}\right)+2 \tau \delta_{I}^{\prime}\left(t_{k}\right)+2 \tau^{2} \delta_{I}^{\prime \prime}\left(t_{k}\right)\right)+O\left(\tau^{3}\right) \\
= & \delta_{I}\left(t_{k}\right)+\left(\beta_{1}+2 \beta_{2}\right) \tau \delta_{I}^{\prime}\left(t_{k}\right)+\frac{1}{2}\left(\beta_{1}+4 \beta_{2}\right) \tau^{2} \delta_{I}^{\prime \prime}\left(t_{k}\right)+O\left(\tau^{3}\right) .
\end{aligned}
$$

Since $\delta_{I}\left(t_{k}\right)=O\left(\tau^{2}\right)$, it follows that

$$
\widehat{\varepsilon}\left(t_{k}\right)=\delta_{I}\left(t_{k}\right)-\sigma(E) \delta_{I}\left(t_{k}\right)=O\left(\tau^{3}\right) .
$$

In a similar way, a Taylor expansion of $\delta_{I}$ at $t=t_{k+1}$ and $t=t_{k+2}$ around $t_{k}$ gives, because of $\rho(1)=0$,

$$
\begin{aligned}
\rho(E) \delta_{I}\left(t_{k}\right) & =\left(\alpha_{0}+\alpha_{1}+\alpha_{2}\right) \delta_{I}\left(t_{k}\right)+\left(\alpha_{1}+2 \alpha_{2}\right) \tau \delta_{I}^{\prime}\left(t_{k}\right)+\frac{1}{2}\left(\alpha_{1}+4 \alpha_{2}\right) \tau^{2} \delta_{I}^{\prime \prime}\left(t_{k}\right)+O\left(\tau^{3}\right) \\
& =-\tau\left(\alpha_{1}+2 \alpha_{2}\right) \delta_{I}^{\prime}\left(t_{k}\right)-\frac{1}{2}\left(\alpha_{1}+4 \alpha_{2}\right) \tau^{2} \delta_{I}^{\prime \prime}\left(t_{k}\right)+O\left(\tau^{3}\right)=O\left(\tau^{3}\right) .
\end{aligned}
$$

Because of $\delta_{D}(t)=O\left(\tau^{3}\right)$ (see Section 3.1) we infer that

$$
\widehat{\delta}\left(t_{k}\right)=\delta_{D}\left(t_{k}\right)-\rho(E) \delta_{I}\left(t_{k}\right)=O\left(\tau^{3}\right) .
$$

Concerning the first time step of the scheme, we observe that, by a Taylor expansion,

$$
\tau v_{t}\left(t_{1}\right)=v\left(t_{1}\right)-v\left(t_{0}\right)+f_{0}, \quad\left\|f_{0}\right\| \leq \frac{\tau^{2}}{2}\left\|v_{t t}\right\|_{L^{\infty}(0, T ; \mathcal{H})}
$$

and (44) becomes

$$
v\left(t_{1}\right)-v\left(t_{0}\right)+\tau B\left(v\left(t_{1}\right)\right)=-f_{0} .
$$

The difference of scheme (21), formulated as $\left(v_{1}-v_{0}\right)+\tau B\left(v_{1}\right)=0$, and (48) becomes

$$
e_{1}-e_{0}+\tau\left(B\left(v_{1}\right)-B\left(v\left(t_{1}\right)\right)=f_{0},\right.
$$


where $e_{j}=v_{j}-v\left(t_{j}\right), j=0,1$. Then, taking this equation in the scalar product with $e_{1}$ and employing $e_{0}=0$ and the one-sided Lipschitz condition of $B$, we obtain

$$
\left\|e_{1}\right\|^{2} \leq \kappa_{1} \tau\left\|e_{1}\right\|^{2}+\left(f_{0}, e_{1}\right) \leq \kappa_{1} \tau\left\|e_{1}\right\|^{2}+\frac{1}{2}\left\|f_{0}\right\|^{2}+\frac{1}{2}\left\|e_{1}\right\|^{2} .
$$

The above error estimate for $f_{0}$ yields $\left\|e_{1}\right\| \leq C \tau^{2}$ if $\tau<1 /\left(2 \kappa_{1}\right)$, where $C>0$ depends on $\left\|v_{t t}\right\|_{L^{\infty}(0, T ; \mathcal{H})}$.

The difference of the equations $\rho(E) v_{k}+\tau B\left(\sigma(E) v_{k}\right)=0$ and (45) leads to the error equations for $e_{k}=v_{k}-\widehat{v}\left(t_{k}\right)(k \geq 2)$ :

$$
\rho(E) e_{k}+\tau\left(B\left(\sigma(E) v_{k}\right)-B\left(\sigma(E) \widehat{v}\left(t_{k}\right)-\widehat{\varepsilon}\left(t_{k}\right)\right)\right)=-\widehat{\delta}\left(t_{k}\right), \quad k \geq 0 .
$$

Taking these equations in the dual product with $\sigma(E) e_{k}+\widehat{\varepsilon}\left(t_{k}\right)$ gives

$$
\begin{aligned}
\left(\rho(E) e_{k}, \sigma(E) e_{k}\right)= & -\tau\left\langle B\left(\sigma(E) v_{k}\right)-B\left(\sigma(E) \widehat{v}\left(t_{k}\right)-\widehat{\varepsilon}\left(t_{k}\right)\right), \sigma(E) e_{k}+\widehat{\varepsilon}\left(t_{k}\right)\right\rangle \\
& -\left(\rho(E) e_{k}, \widehat{\varepsilon}\left(t_{k}\right)\right)-\left(\widehat{\delta}\left(t_{k}\right), \sigma(E) e_{k}+\widehat{\varepsilon}\left(t_{k}\right)\right) .
\end{aligned}
$$

We estimate these expressions term by term. By the G-stability, the left-hand side becomes

$$
\left(\rho(E) e_{k}, \sigma(E) e_{k}\right) \geq \frac{1}{2}\left\|E_{k+1}\right\|_{G}^{2}-\frac{1}{2}\left\|E_{k}\right\|_{G}^{2},
$$

where $E_{k}=\left(e_{k}, e_{k+1}\right)$. With the one-sided Lipschitz condition for $B$ and the CauchySchwarz inequality, it follows for the first term of the right-hand side of (49) that

$$
\begin{aligned}
& -\tau\left\langle B\left(\sigma(E) v_{k}\right)-B\left(\sigma(E) \widehat{v}\left(t_{k}\right)+\widehat{\varepsilon}\left(t_{k}\right)\right), \sigma(E) e_{k}+\widehat{\varepsilon}\left(t_{k}\right)\right\rangle \\
& \leq \kappa_{1} \tau\left\|\sigma(E) e_{k}+\widehat{\varepsilon}\left(t_{k}\right)\right\|^{2} \leq 2 \kappa_{1} \tau\left\|\sigma(E) e_{k}\right\|^{2}+2 \kappa_{1} \tau\left\|\widehat{\varepsilon}\left(t_{k}\right)\right\|^{2} .
\end{aligned}
$$

Because of the positive definiteness of the matrix $G$, we conclude that $\left\|E_{k}\right\|_{G}^{2} \geq C\left(\left\|e_{k}\right\|^{2}+\right.$ $\left\|e_{k+1}\right\|^{2}$ ) which implies that, for some $C>0$ which depends on $\beta_{j}$ and $G$,

$$
\left\|\sigma(E) e_{k}\right\|^{2} \leq C\left(\left\|e_{k}\right\|^{2}+\left\|e_{k+1}\right\|^{2}+\left\|e_{k+2}\right\|^{2}\right) \leq C\left(\left\|E_{k}\right\|_{G}^{2}+\left\|E_{k+1}\right\|_{G}^{2}\right) .
$$

Then, using (46), we obtain

$$
-\tau\left\langle B\left(\sigma(E) v_{k}\right)-B\left(\sigma(E) \widehat{v}\left(t_{k}\right)+\widehat{\varepsilon}\left(t_{k}\right)\right), \sigma(E) e_{k}+\widehat{\varepsilon}\left(t_{k}\right)\right\rangle \leq C \tau\left(\left\|E_{k}\right\|_{G}^{2}+\left\|E_{k+1}\right\|_{G}^{2}\right)+C \tau^{7},
$$

where here and in the following, $C>0$ denotes a generic constant independent of $k$ and $\tau$. In a similar way, the second term on the right-hand side of (49) is estimated by

$$
-\left(\rho(E) e_{k}, \widehat{\varepsilon}\left(t_{k}\right)\right) \leq \frac{\tau}{2}\left\|\rho(E) e_{k}\right\|^{2}+\frac{1}{2 \tau}\left\|\widehat{\varepsilon}\left(t_{k}\right)\right\|^{2} \leq C \tau\left(\left\|E_{k}\right\|_{G}^{2}+\left\|E_{k+1}\right\|_{G}^{2}\right)+C \tau^{5} .
$$

Finally, we use (46) and (47) to estimate the last term in (49):

$$
\begin{aligned}
-\left(\widehat{\delta}\left(t_{k}\right), \sigma(E) e_{k}+\widehat{\varepsilon}\left(t_{k}\right)\right) & \leq \frac{1}{2 \tau}\left\|\widehat{\delta}\left(t_{k}\right)\right\|^{2}+\frac{\tau}{2}\left\|\sigma(E) e_{k}\right\|^{2}+\left\|\widehat{\delta}\left(t_{k}\right)\right\|\left\|\widehat{\varepsilon}\left(t_{k}\right)\right\| \\
& \leq C \tau\left(\left\|E_{k}\right\|_{G}^{2}+\left\|E_{k+1}\right\|_{G}^{2}\right)+C \tau^{5} .
\end{aligned}
$$

We summarize the above estimates:

$$
\frac{1}{2}\left\|E_{k+1}\right\|_{G}^{2}-\frac{1}{2}\left\|E_{k}\right\|_{G}^{2} \leq C_{1} \tau\left(\left\|E_{k}\right\|_{G}^{2}+\left\|E_{k+1}\right\|_{G}^{2}\right)+C_{2} \tau^{5} .
$$


Since $(1+x) /(1-x) \leq 1+4 x$ for $0 \leq x \leq 1 / 2$, we infer that for $\tau \leq 1 /\left(4 C_{1}\right)$,

$$
\left\|E_{k+1}\right\|_{G}^{2} \leq \frac{1+2 C_{1} \tau}{1-2 C_{1} \tau}\left\|E_{k}\right\|_{G}^{2}+\frac{2 C_{2} \tau^{5}}{1-2 C_{1} \tau} \leq\left(1+8 C_{1} \tau\right)\left\|E_{k}\right\|_{G}^{2}+4 C_{2} \tau^{5} .
$$

Solving these recursive inequalities, it follows that

$$
\begin{aligned}
\left\|E_{k+1}\right\|_{G}^{2} & \leq\left(1+8 C_{1} \tau\right)^{k+1}\left\|E_{0}\right\|_{G}^{2}+4 C_{2} \tau^{5} \sum_{j=0}^{k}\left(1+8 C_{1} \tau\right)^{j} \\
& =\left(1+8 C_{1} \tau\right)^{t_{k+1} / \tau}\left\|E_{0}\right\|_{G}^{2}+\frac{C_{2} \tau^{4}}{2 C_{1}}\left(\left(1+8 C_{1} \tau\right)^{t_{k+1} / \tau}-1\right) \\
& \leq e^{8 C_{1} t_{k+1}}\left\|E_{0}\right\|_{G}^{2}+\frac{C_{2}}{2 C_{1}} e^{8 C_{1} t_{k+1}} \tau^{4} \leq C \tau^{4},
\end{aligned}
$$

where we have used that $\left\|E_{0}\right\|_{G} \leq C\left(\left\|e_{0}\right\|+\left\|e_{1}\right\|\right)=C\left\|e_{1}\right\| \leq C \tau^{2}$. Therefore, $\left\|e_{k}\right\|^{2}+$ $\left\|e_{k+1}\right\|^{2} \leq C\left\|E_{k}\right\|^{2} \leq C \tau^{4}$. Finally, taking into account (47), we find that

$$
\left\|v_{k}-v\left(t_{k}\right)\right\|=\left\|e_{k}-\delta_{I}\left(t_{k}\right)\right\| \leq\left\|e_{k}\right\|+\left\|\delta_{I}\left(t_{k}\right)\right\| \leq C \tau^{2}, \quad k \geq 2 .
$$

This finishes the proof.

\section{NumERICAL EXAMPLES}

In this section, we present some numerical examples for the spatial one-dimensional Shigesada-Kawasaki-Teramoto cross-diffusion system, which illustrate the time decay rate of the entropy functional. Numerical examples for the quantum diffusion equation can be found in [4]. We choose the two-step BDF and $\gamma$-method in time, defined in Remark 4, and finite differences in space.

The grid is defined by $x_{i}=i h, i=0, \ldots, N$, and $t_{k}=k \tau, k \geq 0$, with constant space step size $h=1 / N>0$ and time step size $\tau>0$. In the numerical simulations, we have taken $h=0.005$ and $\tau=10^{-6}$. We choose the initial datum $u^{(1)}(x, 0)=2 e^{-x} \sin (2 \pi x)+10$ and $u^{(2)}(x, 0)=-4 e^{-x} \sin (2 \pi x)+10$ for $x \in(0,1)$.

The operator $A=\left(A_{1}, A_{2}\right)$ is discretized in its formulation $A_{j}(u)=-\operatorname{div}\left(d_{j} u^{(j)}+\right.$ $\frac{1}{2} a_{j} \nabla\left(u^{(j)}\right)^{2}+\nabla\left(u^{(1)} u^{(2)}\right)$, where $j=1,2$. Then the two-step BDF (or simpler BDF2) scheme for $v_{1, i}^{k}$, approximating $u^{(1)}\left(x_{i}, t_{k}\right)^{\alpha / 2}$, and $v_{2, i}^{k}$, approximating $u^{(2)}\left(x_{i}, t_{k}\right)^{\alpha / 2}$, reads as follows:

$$
\begin{aligned}
\frac{2}{\alpha}\left(v_{1, i}^{k}\right)^{\alpha / 2-1} & \left(\frac{3}{2} v_{1, i}^{k}-2 v_{1, i}^{k-1}+\frac{1}{2} v_{1, i}^{k-2}\right)-\frac{d_{1} \tau}{h^{2}}\left(\left(v_{1, i+1}^{k}\right)^{\alpha / 2}-2\left(v_{1, i}^{k}\right)^{\alpha / 2}+\left(v_{1, i-1}^{k}\right)^{\alpha / 2}\right) \\
& -\frac{a_{1} \tau}{2 h^{2}}\left(\left(v_{1, i+1}^{k}\right)^{\alpha}-2\left(v_{1, i}^{k}\right)^{\alpha}+\left(v_{1, i-1}^{k}\right)^{\alpha}\right) \\
& -\frac{\tau}{h^{2}}\left(\left(v_{1, i+1}^{k} v_{2, i+1}^{k}\right)^{\alpha / 2}-2\left(v_{1, i}^{k} v_{2, i}^{k}\right)^{\alpha / 2}+\left(v_{1, i-1}^{k} v_{2, i-1}^{k}\right)^{\alpha / 2}\right)=0, \\
\frac{2}{\alpha}\left(v_{2, i}^{k}\right)^{\alpha / 2-1} & \left(\frac{3}{2} v_{2, i}^{k}-2 v_{2, i}^{k-1}+\frac{1}{2} v_{2, i}^{k-2}\right)-\frac{d_{2} \tau}{h^{2}}\left(\left(v_{2, i+1}^{k}\right)^{\alpha / 2}-2\left(v_{2, i}^{k}\right)^{\alpha / 2}+\left(v_{1, i-1}^{k}\right)^{\alpha / 2}\right) \\
& -\frac{a_{2} \tau}{2 h^{2}}\left(\left(v_{2, i+1}^{k}\right)^{\alpha}-2\left(v_{2, i}^{k}\right)^{\alpha}+\left(v_{1, i-1}^{k}\right)^{\alpha}\right)
\end{aligned}
$$



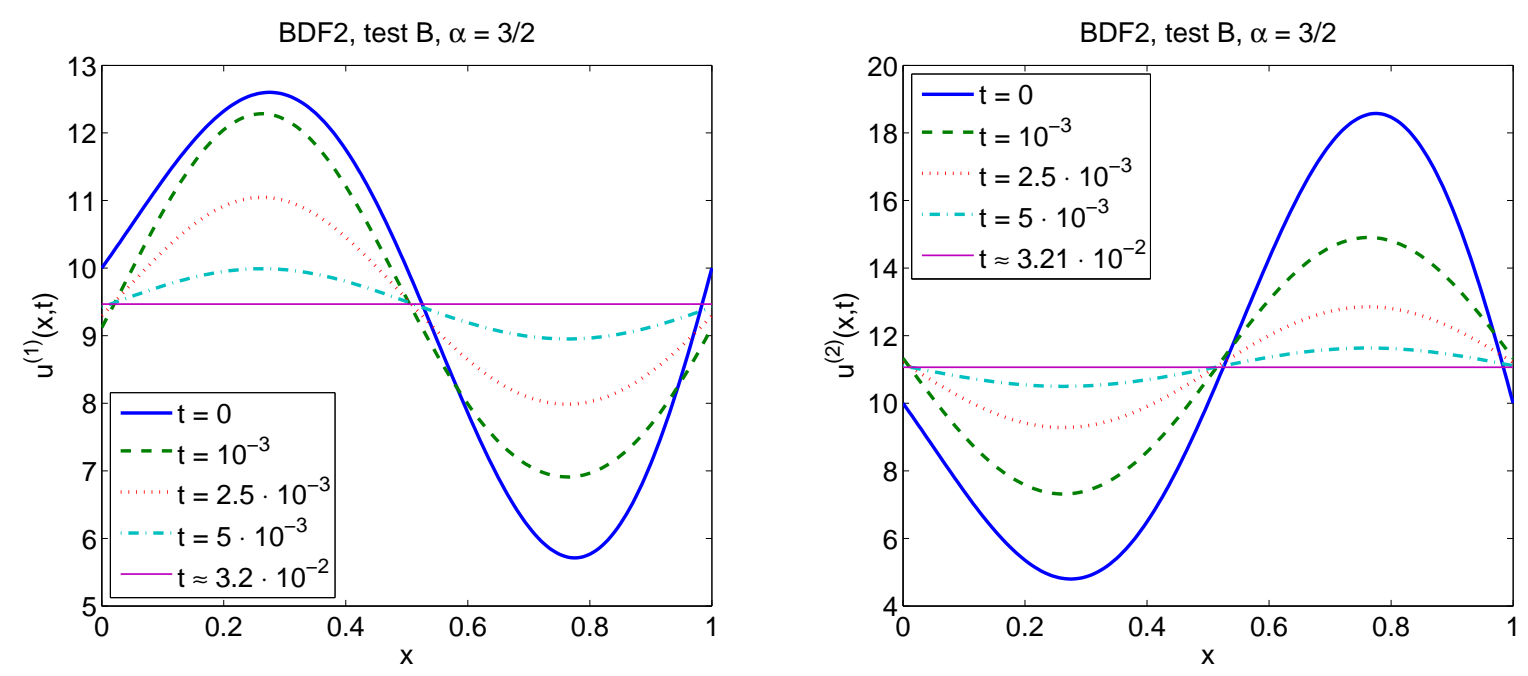

Figure 1. Time evolution of the solution $u^{(1)}(x, t)$ and $u^{(2)}(x, t)$ to the population model computed from the BDF2 scheme (Test B, $\alpha=3 / 2$ ).

$$
-\frac{\tau}{h^{2}}\left(\left(v_{1, i+1}^{k} v_{2, i+1}^{k}\right)^{\alpha / 2}-2\left(v_{1, i}^{k} v_{2, i}^{k}\right)^{\alpha / 2}+\left(v_{1, i-1}^{k} v_{2, i-1}^{k}\right)^{\alpha / 2}\right)=0
$$

where $i=1, \ldots, N-1$ and $k \geq 2$. To determine the discrete solution for $k=1$, we employ the implicit Euler method. The periodic boundary conditions are $v_{j, 0}=v_{j, N}$ and $v_{j, 1}=v_{j, N+1}$ for $j=1,2$. The above nonlinear system is solved by the Newton method. The scheme using the $\gamma$-method (with $\gamma=1 / 5$ ) is defined in a similar way. The parameters are chosen as follows:

$$
\text { Test A: } d_{1}=d_{2}=1, a_{1}=a_{2}=0.01, \quad \text { Test B: } d_{1}=d_{2}=1, a_{1}=a_{2}=1 .
$$

In Test $\mathrm{A}$, the self-diffusion parameters are small compared to the remaining terms, whereas in Test B, all parameters, including the cross-diffusion terms, are of the same order.

In Figure 1, the time evolution of the population densities $u^{(1)}$ and $u^{(2)}$ for the parameters according to Test B, $\alpha=3 / 2$, computed from the BDF2 scheme, is illustrated. Because of the absence of source terms and the periodic boundary conditions, the densities converge to the constant steady state for large times.

The convergence of the scheme is shown in Figure 2 at time $t_{m}=5 \cdot 10^{-4}$. The error is measured in the $\ell^{2}$ norm

$$
\left\|e_{m}\right\|_{2}=\left(\sum_{i=0}^{N-1} \sum_{j=1}^{2}\left(v_{j, i}^{m}-V_{j, i}^{m}\right)^{2} h\right)^{1 / 2}
$$

where $V_{j, i}^{m}$ is the reference solution computed by using the very small time step $\tau=10^{-8}$. The rates have been obtained by the linear regression method. As expected, the rate of convergence is (approximately) two, even for $\alpha=1$ which was excluded in our analysis. The rate for $\alpha=2$ is the largest which comes from the fact that in this case, we recover the usual BDF2 method without additional nonlinearities (since $w_{k}=v_{k}$ ). 


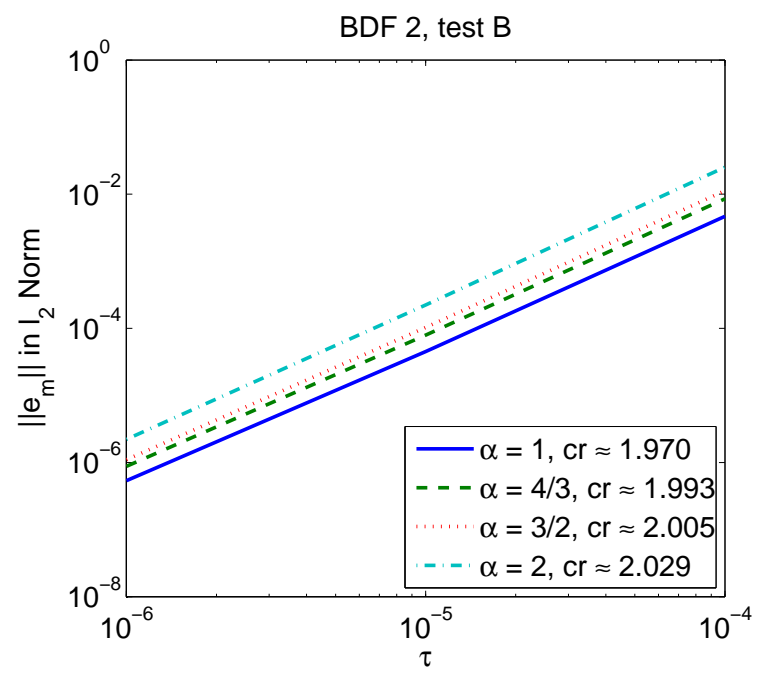

Figure 2. Error $\left\|e_{m}\right\|_{2}$ versus the time step size for the BDF2 scheme at time $t_{m}=5 \cdot 10^{-4}$.
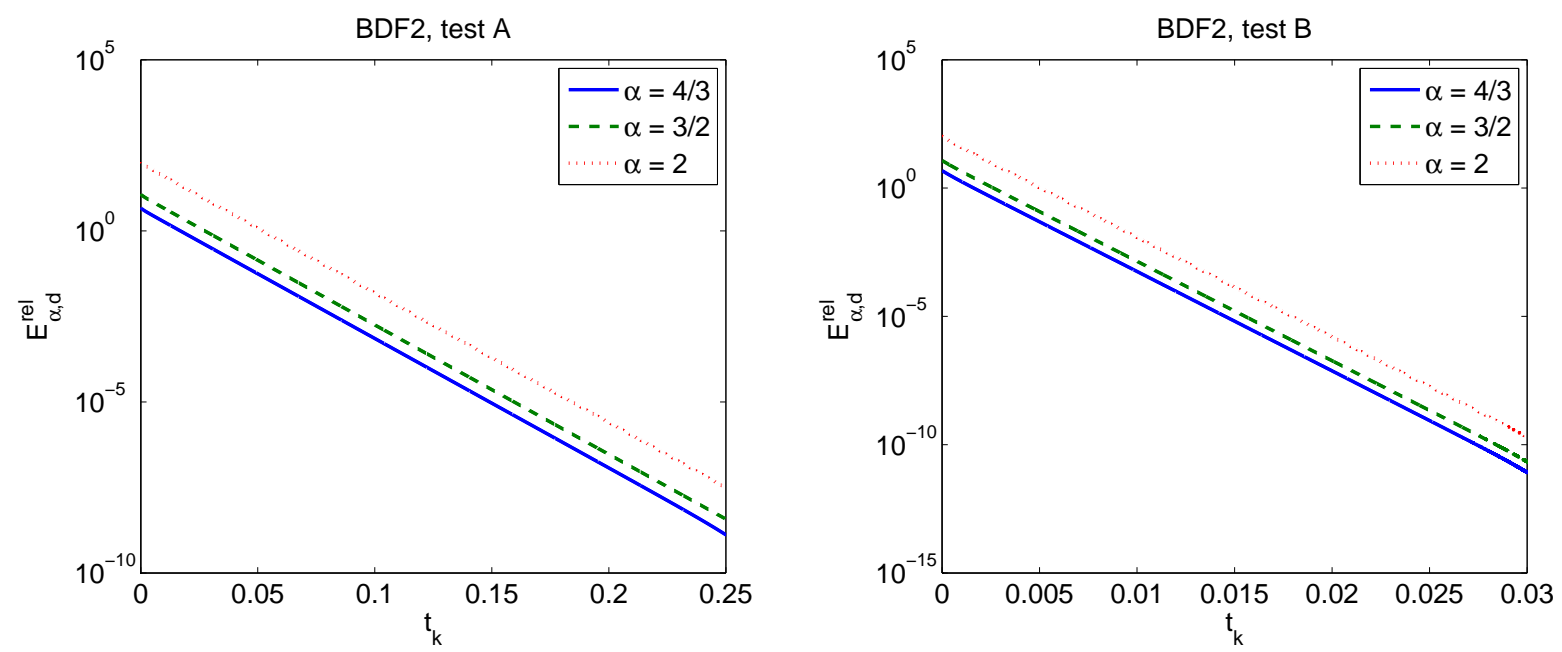

FiguRE 3. The relative entropy for the BDF2 scheme versus time.

Figures 3 and 4 illustrate the time decay of the discrete relative entropy $E_{\alpha, d}^{\mathrm{rel}}=H_{\alpha, d}\left[V_{k}\right]-$ $H_{\alpha, d}\left[V^{*}\right]$, where

$$
H_{\alpha, d}\left[V_{k}\right]=\frac{1}{2} \sum_{i, j=0}^{1} G_{i j} \sum_{\ell=1}^{N-1}\left(v_{1, \ell}^{k+i} v_{1, \ell}^{k+j}+v_{2, \ell}^{k+i} v_{2, \ell}^{k+j}\right),
$$

and $V^{*}$ represents the (constant) stationary solution. The coefficients of the matrix $\left(G_{i j}\right)$ are given in Remark 4. We observe that in all considered cases, the discrete entropy converges to the equilibrium with exponential rate. 

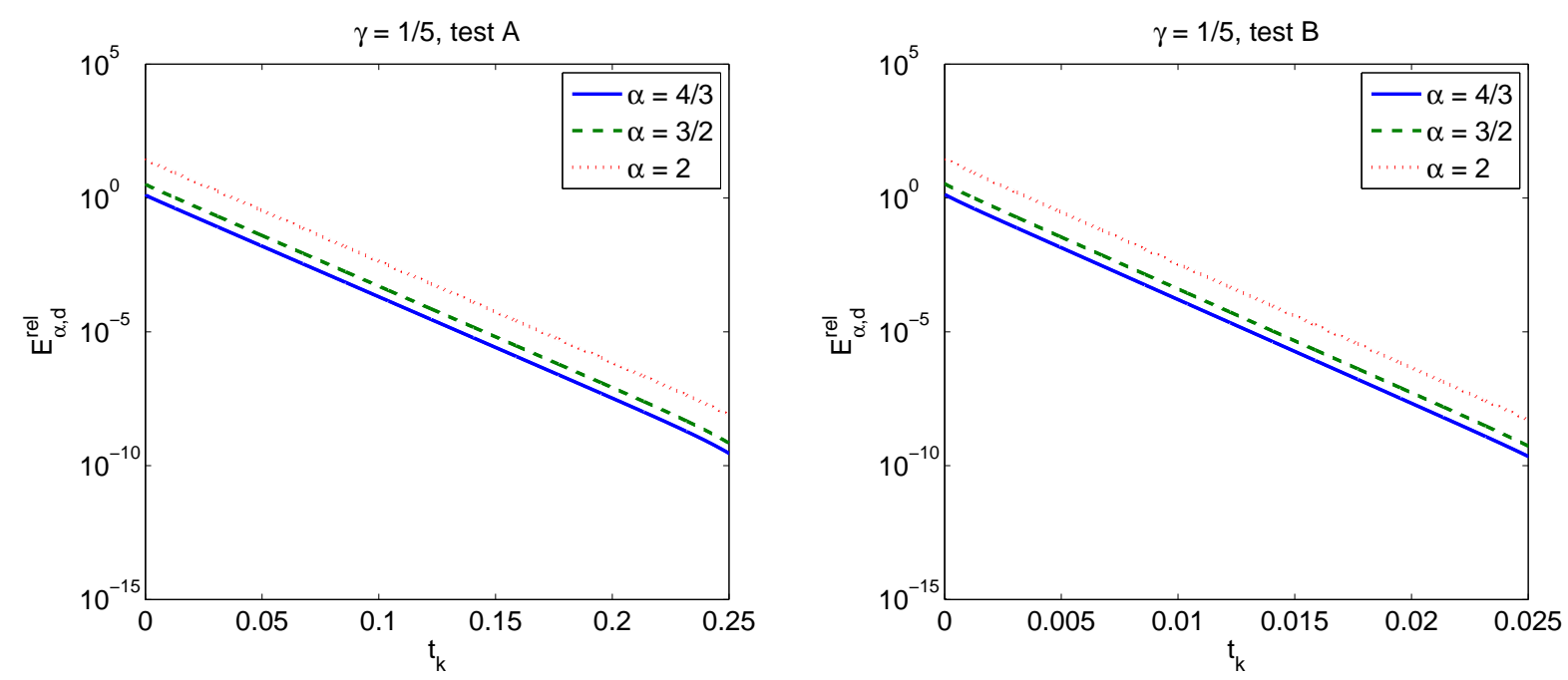

FigurE 4. The relative entropy for the $\gamma$-method versus time $(\gamma=1 / 5)$.

\section{Appendix A. A family of SECOnd-ORDER G-STABle one-LEG Methods}

We derive all G-stable one-leg schemes which are of second order (in the truncation error). Then $p=2$ and for $v=\left(v_{0}, v_{1}, v_{2}\right)^{\top}$,

$$
\rho(E) v=\alpha_{0} v_{0}+\alpha_{1} v_{1}+\alpha_{2} v_{2}, \quad \sigma(E) v=\beta_{0} v_{0}+\beta_{1} v_{1}+\beta_{2} v_{2} .
$$

The normalization, consistency, and second-order accurate conditions (see Section 3.1) lead to the four equations

$$
\begin{array}{ll}
1=\sigma(1)=\beta_{0}+\beta_{1}+\beta_{2}, & 0=\rho(1)=\alpha_{0}+\alpha_{1}+\alpha_{2}, \\
1=\sigma(1)=\rho^{\prime}(1)=\alpha_{1}+2 \alpha_{2}, & 1+2 \alpha_{2}=\rho^{\prime}(1)+\rho^{\prime \prime}(1)=2 \sigma^{\prime}(1)=2 \beta_{1}+4 \beta_{2} .
\end{array}
$$

The G-stability condition (25) can be written as

$$
\rho(E) v \sigma(E) v-\frac{1}{2} V_{1}^{\top} G V_{1}+\frac{1}{2} V_{0}^{\top} G V_{0}-\left(\gamma_{0} v_{0}+\gamma_{1} v_{1}+\gamma_{2} v_{2}\right)^{2}=0
$$

where $V_{1}=\left(v_{1}, v_{2}\right)^{\top}, V_{0}=\left(v_{0}, v_{1}\right)^{\top}$, and $\gamma_{j}$ are some real constants. This formulation is possible since the occuring polynomials are at most quadratic and consequently, the positive polynomial $p(v)=\rho(E) v \sigma(E) v-\frac{1}{2} V_{1}^{\top} G V_{1}+\frac{1}{2} V_{0}^{\top} G V_{0}$ can be written as a single square. Condition (52) has to hold for all $v \in \mathbb{R}^{3}$. Identifying the coefficients of $p(v)$ with those from $\left(\gamma_{0} v_{0}+\gamma_{1} v_{1}+\gamma_{2} v_{2}\right)^{2}$, we find the following six equations:

$$
\begin{array}{ll}
0=\alpha_{0} \beta_{0}+G_{00}-\gamma_{0}^{2}, & 0=2 G_{01}+\alpha_{1} \beta_{0}+\alpha_{0} \beta_{1}- \\
0=\alpha_{0} \beta_{2}+\alpha_{2} \beta_{0}-2 \gamma_{0} \gamma_{2}, & 0=\gamma_{11}-\gamma_{11}+\alpha_{1} \beta_{1}-\gamma_{1}^{2}, \\
0=-2 G_{01}+\alpha_{2} \beta_{1}+\alpha_{1} \beta_{2}-2 \gamma_{1} \gamma_{2}, & 0=-G_{11}+\alpha_{2} \beta_{2}-\gamma_{2}^{2},
\end{array}
$$

where $G=\left(G_{i j}\right)_{i, j=0,1}$. Observing that $G_{01}=G_{10}$, conditions (51) and (53) yield 10 equations for the 12 unknowns $G_{00}, G_{01}, G_{11}, \alpha_{j}, \beta_{j}, \gamma_{j}(i, j=0,1,2)$. We also require the 
positive definiteness of the matrix $G$, i.e.

$$
G_{00}>0, \quad \operatorname{det} G=G_{00} G_{11}-G_{01}^{2}>0 .
$$

Solving the nonlinear system (51) and (53) with the command solve in Maple gives two sets of solutions. One solution set yields a matrix $G$ with $\operatorname{det} G=0$ such that this solution can be excluded. The other set is given by

$$
\left(\alpha_{0}, \alpha_{1}, \alpha_{2}\right)=\left(\alpha_{2}-1,1-2 \alpha_{2}, \alpha_{2}\right), \quad\left(\beta_{0}, \beta_{1}, \beta_{2}\right)=\left(\frac{1}{2}-\alpha_{2}+\beta_{2}, \frac{1}{2}+\alpha_{2}-2 \beta_{2}, \beta_{2}\right),
$$

where $\alpha_{2}$ and $\beta_{2}$ are free parameters, the matrix

$$
G=\frac{1}{4}\left(\begin{array}{cc}
\left(2 \alpha_{2}-5\right) \alpha_{2}+2 \beta_{2}+2 & \left(-2 \alpha_{2}+3\right) \alpha_{2}-2 \beta_{2} \\
\left(-2 \alpha_{2}+3\right) \alpha_{2}-2 \beta_{2} & \left(2 \alpha_{2}-1\right) \alpha_{2}+2 \beta_{2}
\end{array}\right),
$$

and the constants $\gamma_{j}$ are solutions of certain quadratic equations involving the coefficients $\alpha_{j}$ and $\beta_{j}$. The matrix $G$ is positive definite if and only if $\left(2 \alpha_{2}-5\right) \alpha_{2}+2 \beta_{2}+2>0$ and $\operatorname{det} G=\beta_{2}-\alpha_{2} / 2>0$. The latter condition implies the former one since

$$
\left(2 \alpha_{2}-5\right) \alpha_{2}+2 \beta_{2}+2>\left(2 \alpha_{2}-5\right) \alpha_{2}+\alpha_{2}+2=2\left(\alpha_{2}-1\right)^{2} \geq 0 .
$$

Consequently, all one-leg schemes (54) satisfying $\beta_{2}>\alpha_{2} / 2$ are of second order and Gstable.

The examples given in Remark 4 are included in the above family of schemes. Indeed, choosing $\alpha_{2}=\frac{3}{2}$ and $\beta_{2}=1$, we find the two-step BDF method, and setting for $\gamma \in(0,1]$

$\alpha_{2}=1 /(\gamma+1)$ and $\beta_{2}=(3 \gamma+1) /\left(2(\gamma+1)^{2}\right)$, we recover the $\gamma$-method of [12, 35].

Notice that we could repeat the same procedure to derive all first-order G-stable schemes. The solution set will depend on three free parameters since only 9 equations for 12 unknowns need to be solved. We leave the details to the reader.

\section{REFERENCES}

[1] B. Andreianov, M. Bendahmane, and R. R. Baier. Analysis of a finite volume method for a crossdiffusion model in population dynamics. Math. Models Meth. Appl. Sci. 21 (2011), 307-344.

[2] C. Baiocchi and M. Crouzeix. On the equivalence of A-stability and G-stability. Appl. Numer. Math. 5 (1989), 19-22.

[3] J. Barrett and J. Blowey. Finite element approximation of a nonlinear cross-diffusion population model. Numer. Math. 98 (2004), 195-221.

[4] M. Bukal, E. Emmrich, and A. Jüngel. Entropy-stable and entropy-dissipative approximations of a fourth-order quantum diffusion equation. To appear in Numer. Math., 2013. arXiv:1208.5474.

[5] M. Bukal, A. Jüngel, and D. Matthes. A multidimensional nonlinear sixth-order quantum diffusion equation. Ann. Inst. H. Poincaré Anal. non lin. 30 (2013), 337-365.

[6] J. A. Carrillo, A. Jüngel, and S. Tang. Positive entropic schemes for a nonlinear fourth-order equation. Discrete Contin. Dyn. Sys. B 3 (2003), 1-20.

[7] L. Chen and A. Jüngel. Analysis of a multi-dimensional parabolic population model with strong cross-diffusion. SIAM J. Math. Anal. 36 (2004), 301-322.

[8] L. Chen and A. Jüngel. Analysis of a parabolic cross-diffusion population model without self-diffusion. J. Diff. Eqs. 224 (2006), 39-59.

[9] G. Dahlquist. A special stability problem for linear multistep methods. BIT 3 (1963), 27-43. 
[10] G. Dahlquist. Error analysis for a class of methods for stiff nonlinear initial value problems. Proc. Numer. Anal. Conf., Dundee, Scotland, 1975. Lecture Notes Math. 506, pp. 60-74. Springer, New York, 1976.

[11] G. Dahlquist. G-stability is equivalent to A-stability. BIT 18 (1978), 384-401.

[12] G. Dahlquist, W. Liniger, and O. Nevanlinna. Stability of two-step methods for variable integration steps. SIAM J. Numer. Anal. 20 (1983), 1071-1085.

[13] R. Dal Passo, H. Garcke, and G. Grün. On a fourth-order degenerate parabolic equation: global entropy estimates, existence, and qualitative behavior of solutions. SIAM J. Math. Anal. 29 (1998), 321-342.

[14] P. Degond, F. Méhats, and C. Ringhofer. Quantum energy-transport and drift-diffusion models. J. Stat. Phys. 118 (2005), 625-665.

[15] B. Derrida, J. Lebowitz, E. Speer, and H. Spohn. Fluctuations of a stationary nonequilibrium interface. Phys. Rev. Lett. 67 (1991), 165-168.

[16] B. Düring, D. Matthes, and J.-P. Milišić. A gradient flow scheme for nonlinear fourth order equations. Discrete Contin. Dyn. Sys. B 14 (2010), 935-959.

[17] E. Emmrich. Stability and error of the variable two-step BDF for semilinear parabolic problems. $J$. Appl. Math. Comput. 19 (2005), 33-55.

[18] E. Emmrich. Two-step BDF time discretization of nonlinear evolution problems governed by monotone operators with strongly continuous perturbations. Comput. Meth. Appl. Math. 9 (2009), 37-62.

[19] E. Emmrich and M. Thalhammer. Stiffly accurate Runge-Kutta methods for nonlinear evolution problems governed by a monotone operator. Math. Comp. 79 (2010), 785-806.

[20] G. Galiano, M. L. Garzòn, and A. Jüngel. Semi-discretization and numerical convergence of a nonlinear cross-diffusion population model. Numer. Math. 93 (2003), 655-673.

[21] G. Gambino, M. C. Lombardo, and M. Sammartino. A velocity-diffusion method for a Lotka-Volterra system with nonlinear cross and self-diffusion. Appl. Numer. Math. 59 (2009), 1059-1074.

[22] U. Gianazza, G. Savaré, and G. Toscani. The Wasserstein gradient flow of the Fisher information and the quantum drift-diffusion equation. Arch. Ration. Mech. Anal. 194 (2009), 133-220.

[23] E. Hansen. Convergence of multistep time discretizations of nonlinear dissipative evolution equations. SIAM J. Numer. Anal. 44 (2006), 55-65.

[24] C. González, A. Ostermann, C. Palencia, and M. Thalhammer. Backward Euler discretization of fully nonlinear parabolic problems. Math. Comp. 71 (2002), 125-145.

[25] E. Hairer and G. Wanner. Solving Ordinary Differential Equations II. Stiff and Differential-Algebraic Problems. Springer, Berlin, 1991.

[26] A. Hill. Global dissipativity for A-stable methods. SIAM J. Numer. Anal. 34 (1997), 119-142.

[27] C. Huang. Dissipativity of one-leg methods for dynamical systems with delays. Appl. Numer. Math. 35 (2000), 11-22.

[28] W. Hundsdorfer and B. Steininger. Convergence of linear multistep and one-leg methods for stiff nonlinear initial value problems. BIT 31 (1991), 124-143.

[29] A. Jüngel. Transport Equations for Semiconductors. Lecture Notes in Physics 773, Springer, Berlin, 2009.

[30] A. Jüngel and D. Matthes. An algorithmic construction of entropies in higher-order nonlinear PDEs. Nonlinearity 19 (2006), 633-659.

[31] A. Jüngel and D. Matthes. The Derrida-Lebowitz-Speer-Spohn equation: existence, non-uniqueness, and decay rates of the solutions. SIAM J. Math. Anal. 39 (2008), 1996-2015.

[32] A. Jüngel and J.-P. Milišić. A sixth-order nonlinear parabolic equation for quantum systems. SIAM J. Math. Anal. 41 (2009), 1472-1490.

[33] A. Jüngel and R. Pinnau. A positivity preserving numerical scheme for a nonlinear fourth-order parabolic equation. SIAM J. Numer. Anal. 39 (2001), 385-406.

[34] A. Jüngel and R. Pinnau. Convergent semidiscretization of a nonlinear fourth order parabolic system. M2AN Math. Model. Numer. Anal. 37 (2003), 277-289. 
[35] G. Kulikov and S. Shindin. One-leg integration of ordinary differential equations with global error control. Comput. Meth. Appl. Math. 5 (2005), 86-96.

[36] M.-N. Le Roux. Méthodes multipas pour des équations paraboliques non linéaires. Numer. Math. 35 (1980), 143-162.

[37] J. Rulla. Error analysis for implicit approximations to solutions to Cauchy problems. SIAM J. Numer. Anal. 33 (1996), 68-87.

[38] A. Ostermann, M. Thalhammer, and G. Kirlinger. Stability of linear multistep methods and applications to nonlinear parabolic problems. Appl. Numer. Math. 48 (2004), 389-407.

[39] N. Shigesada, K. Kawasaki, and E. Teramoto. Spatial segregation of interacting species. J. Theoret. Biol. 79 (1979), 83-99.

[40] D. Willett and J. Wong. On the discrete analogues of some generalizations of Gronwalls inequality. Monatsh. Math. 69 (1965), 362-367.

[41] E. Zeidler. Nonlinear Functional Analysis and Its Applications. Volume II/A. Springer, New York, 1990.

Institute for Analysis and Scientific Computing, Vienna University of Technology, Wiedner Hauptstrasse 8-10, 1040 Wien, Austria

E-mail address: juengel@tuwien.ac.at

Department of Applied Mathematics, Faculty of Electrical Engineering and Computing, University of Zagreb, Unska 3, 10000 Zagreb, Croatia

E-mail address: pina.milisic@fer.hr 